\title{
ORIGINALBEITRAG \\ Simulationsbasierter Ertragswert als Ergänzung zum Verkehrswert
}

\author{
Werner Gleißner • Tobias Just • Endre Kamarás
}

Eingegangen: 8. Juni 2016 / Angenommen: 9. Mai 2017 / Online publiziert: 12. Juni 2017

(C) Der/die Autor(en) 2017. Dieser Artikel ist eine Open-Access-Publikation.

Zusammenfassung Die Begriffe „Immobilienpreis“ und „Immobilienwert“ werden häufig synonym verwendet. Das ist dann gerechtfertigt, wenn der Markt tatsächlich dafür sorgt, dass die realisierten Preise die nutzenstiftenden Werte der Zahler abbilden. Doch die heftigen Preisumschwünge auf Immobilien- und Immobilienkapitalmärkten sowie die Erkenntnisse der Verhaltensökonomie legen die Vermutung nahe, dass es Phasen gibt, in denen die Transaktionspreise eben nicht als nachhaltige Werte im Sinne eines als sicher empfundenen Äquivalents für diskontierte, unsichere Zahlungsüberschüsse in der Zukunft angesehen werden können. In diesem Beitrag zeigen wir, dass es häufig hilfreich ist, neben die gängigen Preisschätzverfahren, Bewertungen zu stellen, die auf der Grundlage von Simulationen die Unsicherheit zukünftiger Zahlungen über ein Risiko-Wert-Modell berücksichtigen. Hierbei wird die Monte-Carlo-Simulation nicht wie bei anderen Arbeiten alleine für Inputvariablen und das Ableiten einer Bandbreite für geschätzte Preise genutzt. Die Ergebnisse der Simulation des Cashflows dienen in unserer Arbeit als „Input“ für die Ableitung risikoadäquater Diskontierungszinssätze und eines darauf basierenden fundamentalen Werts. Auch die Trennung in Simulation des Boden- und Immobilienwertes ist in unserer Vorgehensweise neu. Zudem werden Risikodiversifikationseffekte in einem Portfolio abgebildet. Der Portfoliowert stellt dann nicht die Summe aller einzelnen

\footnotetext{
W. Gleißner $(\bowtie) \cdot$ E. Kamarás

FutureValue Group AG, Obere Gärten 18, 70771 Leinfelden-Echterdingen, Deutschland

E-Mail: fachartikel@FutureValue.de

E. Kamarás

E-Mail: e.kamaras@FutureValue.de

W. Gleißner

TU Dresden, Dresden, Deutschland

T. Just

IREBS Universitat Regensburg, Universitatsstraße 31, 93040 Regensburg, Deutschland E-Mail: tobias.just@irebs.de
} 
Objektwerte dar, sondern bewertet darüber hinaus Diversifikationseffekte. Ohne die Berücksichtigung der Diversifikationseffekte kann es zu erheblichen Fehlbewertungen von Portfolios kommen. Die zentralen Ergebnisse illustrieren wir anhand eines wohnungswirtschaftlichen Beispiels. Ziel dieser neuen Bewertungsmethode ist nicht ein „,besseres“ Preisschätzmodell anstelle der Verkehrswertberechnung vorzustellen, sondern einen intrinsischen Immobilienwert aus spezifischen Annahmen hinsichtlich des Objekts und aus Sicht des Bewerters zu deduzieren. Das Ziel ist nicht, Marktpreise für Immobilien und Immobilienportfolios besser zu treffen als dies von aktuellen Verkehrswertschätzungen gemacht wird, sondern deren „fundamentalen“ Wert zu bestimmen und damit in Kombination von Marktpreisen, bessere Transaktionsentscheidungen zu ermöglichen.

Schlüsselwörter Verkehrswert · Fundamentaler Ertragswert · Unvollkommene Märkte · Risikoaggregation · Monte-Carlo-Simulation · Preisuntergrenze einer Immobilie · Wertbeitrag der Risikodiversifikation · Ertragsrisiko ·

Cashflowvolatilität · Immobilienbewertung

\section{Simulation-based earnings value as a supplement to the market value}

Abstract The terms real estate price and real estate value are very often used interchangeably. As long as the market leads to the situation that realized prices reflect the utility-based value of the payer, this is justifiable. Heavy price changes on real estate - and real estate capital markets - as well as findings from behavioral economics can challenge this assumption and give us reasons to reckon phases were transaction prices cannot be seen as reflecting long-term values, defined as certainty equivalents of discounted, uncertain future payment surplus. With this article, we argue that it makes sense to use simulation-based valuations, taking into account the uncertainty of future payments as part of a risk-value model. Unlike in other publications, Monte-Carlo simulations are not only used for input variables and for deriving band width for estimated prices. The findings of such a simulation of cash flows here are used as "input" for deriving risk adjusted cost of capital rates that can be used for discounting. This can then be used for deriving a fundamental value. This is typical for a risk-oriented valuation method. We also run separate simulations for the value of land and property itself, contrary to other publications. In addition, we also take into account risk diversification effects as part of a portfolio. We do this with the example of a residential object. The value of such a portfolio then is not the sum of all single objects, but it takes into account diversification effects. Not taking into account such diversification effects can lead to drastically biased valuations. We do not aim at getting a "better" model for estimating the transaction price, but we do derive the intrinsic value of the object based on specific assumptions regarding the object and the valuator. This means to derive the "fundamental" value and therefore in combination with market prices, supporting "better" decisions for transactions.

The valuation result is therefore comparatively independent of possible misjudgements by market participants. In this way, the added value of real estate portfolio or asset management can also be calculated. This does not guarantee a higher return, but the likelihood of systematically neglected risks is clearly reduced. 
It is clear that for a simulation-based valuation numerous assumptions are to be made, i. e. band widths of rents, potential vacancy risks etc. Each of these assumptions can be challenged, but the advantages of the simulation-based valuation are not that they are error-free, but rather that they are less likely to be erroneous than simple "one-point estimates" as they are based on intervals. In addition, diversification effects can also be included explicitly. This is why this valuation method unfolds its strengths in coexistence with the determination of market values as it is still important to estimate realistic potential market prices.

With the "Principles for the Valuation of Real Estate" (IDW S 10), the Institute of German Auditors published a valuation standard based on IDW S 1 (see, among others, Kleiber 2012). This standard is based on the DCF approach. According to IDW S 10 paragraph 63, a valuator may choose between different ways for determining risk-adjusted discount rates.

Instead of applying the CAPM, which is generally preferred by the IDW, one can also make use of the approach of a "component-type" derivation with individual risk premiums, which is also preferred in practice. The components are the risk-free base rate (rf), a general property market-specific risk premium, and a property-specific premium (see Möller 2014). The IDW implicitly assumes constant risk premiums over time (Möller 2014 as well as Creutzmann 2013). Möller (2014, p. 205) states a range of 1.0-1.6\% for this general property-market risk premium, not specified by the IDW, in accordance with the standard on "discounted cashflow valuation for residential properties" of the Federal Association of German Housing and Real Estate Companies ("Discounted Cashflow-Bewertungsverfahren für Wohnimmobilien" des Bundesverbandes deutscher Wohnungs- und Immobilienunternehmen). The objectspecific risk premium should reflect individual factors like location or condition of the object, without discussing procedures for object-specific risk analyses for deriving sound risk premiums normally needed.

It is worth mentioning that an explicit and consistent distinction between intrinsic "real estate value" and an estimated market value (i. e., price) is neither made by the IDW S 10, nor by the IDW S 1 .

Investors who have to rely on stable cash flows for their investment decisions cannot avoid using valuation models that represent cash flow risks adequately. Otherwise, in times of overheated markets, overvalued assets are compared with other overvalued assets. And especially for long-term oriented, risk-averse investors, this can lead to misallocating funds. Risk-based values and (estimated) current prices (market values) have to be clearly distinguished. Both are essential but offer different pieces of information.

Coming from this, it should be mentioned, that by slightly modifying the above methodology, ratings can be derived, and this would enable taking the creditor's perspective.

This valuation is not an alternative pricing model competing with the existing market value estimation, but it allows to calculate the value of a real estate object from the perspective of the valuation subject, based on property and subject-specific assumptions. This means that it delivers a more solid calculation of the "fundamental" value of an object, e.g. as part of a portfolio. In combination with (a separate) 
market price estimation, this helps making better decisions with regards to buying and selling property or with regards to structuring real estate portfolios.

Keywords Market value $\cdot$ Fundamental earnings value $\cdot$ Imperfect markets $\cdot$ Risk aggregation · Monte Carlo method · Lower price limit of a property · Value added of risk diversification - Earnings risk · Cash flow volatility $\cdot$ Real estate valuation

\section{Preis und Wert von Immobilien}

Im alltäglichen Sprachgebrauch werden die Begriffe „Preis“ und „Wert“ oft als Synonyme verwendet. Dies ist jedoch fahrlässig, weil es wichtige Unterschiede gibt: Der fundamentale Wert einer Immobilie entspricht dem Geldbetrag, der als sicheres Äquivalent zu den zukünftigen unsicheren Cashflows des Assets gelten kann. ${ }^{1}$ Der Marktpreis hingegen ist das Resultat einer Verkaufstransaktion und bei Immobilien immer das Resultat eines Verhandlungsprozesses. Preise lassen sich beobachten, Werte nicht: Werte können nur mit Hilfe von Modellen approximiert werden, die den Informationsstand, Handlungsalternativen und Präferenzen des Bewerters (Käufer oder Verkäufer) erfassen.

In der Praxis wird unter der Bezeichnung „Immobilienbewertung“ versucht, mögliche Kauf- bzw. Verkaufspreise zu schätzen. Auch die Bewertungsmethoden, wie sie im $§ 194$ BewG (Bewertungsgesetz) kodifiziert sind, lassen sich (näherungsweise) als „Preisschätzverfahren“ auffassen. Auch der sogenannte „Verkehrswert“, wie ihn das Baugesetzbuch ( $\$ 194$ BauGB) kennt, ist letztlich ein geschätzter Verkaufspreis.

Nur in einem vollkommenen Markt stimmen Preise und Werte überein. Weil Immobilienmärkte jedoch zahlreiche Unvollkommenheiten aufweisen - die gehandelten Güter sind inhomogen, die Informationen sind asymmetrisch verteilt, und es gibt erhebliche Transaktionskosten - können Preise und Werte auf Immobilienmärkten auseinanderfallen (vgl. zu den Besonderheiten der Immobilienmärkte u. a. Just und Uttich 2015). Dies zeigt sich in unerwarteten, heftigen Preiskorrekturen wie jenen in den USA, in Spanien oder in Irland nach 2007. Die Anpassungsprozesse sorgten bei Immobilieninvestoren sowie Kreditinstituten für erhebliche Verluste, wenn sie ihre Beleihungsgrenzen eher an den realisierten Preisen und nicht an fundamentalen Werten ausrichteten. Von einer Blase wird dann gesprochen, wenn die Preise sehr weit von den fundamental gerechtfertigten ,Werten“ entfernt sind. Wenn man die Werte aber nicht kennt, lässt sich eine Blase im Diktum von Milton Friedman (1953) schwer ex ante erkennen. Je besser es gelingt, die fundamentalen Werte abzubilden, umso eher lassen sich heftige Korrekturen von Fehlbewertungen reduzieren.

In diesem Beitrag geht es um die Ermittlung eines solchen risikogerechten Wertes mittels Simulationsmodellen und wie diese Werte Immobilienmarktakteuren helfen können, eine Fehlbewertung zu erkennen und so Investitionsstrategien zu konzipieren. Simulationsmodelle finden spätestens seit den wegbereitenden Arbeiten von Hertz (1968), Wofford (1978) sowie später Hughes (1995) auch in der Immobilien-

\footnotetext{
1 Siehe präzisierend Laux und Schabel (2009). Es ist hierbei weitgehend irrelevant, ob ein direktes oder indirektes Immobilieninvestment betrachtet wird.
} 
bewertung ihre Anwendung, um Unsicherheit über die Entwicklung von Wertbestimmungsfaktoren abzubilden. Dass diese Unsicherheit gerade angesichts unzureichender Markttransparenz für Immobilientransaktionen sehr hoch ist, zeigt Pfnür (2002). Noch immer fehlen methodische Standards wie solch eine Risikoberücksichtigung für die Immobilienwirtschaft erfolgen kann. Im Bereich der Unternehmensbewertung ist man hier mit dem Bewertungsstandard der deutschen Wirtschaftsprüfer (IDW S 1) dem Grunde nach weiter, wenngleich die Risikoerfassung in der Praxis auch in der Unternehmensbewertung noch oft über das Capital-Asset-PricingModell (CAPM) erfolgt. Im IDW S 10 zur Immobilienbewertung ist die Abkehr vom CAPM weitgehend vollzogen (vgl. Pohl 2013 sowie Creutzmann 2013). Die Bewertungsverfahren des IDW S 10 zielen dabei eher auf das Schätzen von Transaktionspreisen und weniger auf das Abbilden eines intrinsischen Wertes, der alle zukünftigen Risiken in der Zukunft angemessen diskontiert (IDW 2013). Für solche Risikoschätzungen hat sich in den letzten Jahrzehnten die Monte-Carlo-Simulation als Methode etabliert.

Anders als bisher im Schrifttum wird die Monte-Carlo-Simulation in diesem Beitrag aber nicht genutzt, um Unsicherheit (bzw. Bandbreiten) des geschätzten Preises zu verdeutlichen. Hier dienen die Ergebnisse der Monte-Carlo-Simulation als Input für ein Risiko-Wert-Modell, d. h. die Simulation und die Bewertungstheorie werden verknüpft, und dies erlaubt eine vollständige Unsicherheitstransformation. Dies ist interpretierbar als sicherer Gegenwert für einen unsicheren zukünftigen Cash-Flow einer Immobilie. ${ }^{2}$

Der weitere Beitrag gliedert sich dabei wie folgt: Im Abschn. 2 wird die in der Praxis relevante Vorgehensweise der Immobilienbewertung skizziert. Abschn. 3 kennzeichnet die Idee der risikogerechten Bewertung, und es wird ein Überblick über die Literatur von Simulationen für Immobilienbewertungen gegeben; Abschn. 4 stellt unser Vorgehen einer simulationsbasierten Immobilienbewertung vor. Hier spielen insbesondere die Aspekte der Trennung von Boden- und Immobilienwert sowie das spezifische Risiko-Wert-Modell und die Bedeutung von Diversifikationseffekten in Portfolios eine entscheidende Rolle. Im Abschn. 5 wird mit Hilfe eines Beispiels die Vorgehensweise illustriert. Abschn. 6 fasst die Ergebnisse zusammen.

\section{Bewertungsverfahren in der Praxis}

In der deutschen Immobilienbewertung werden drei Verfahren unterschieden: erstens das Vergleichswertverfahren, bei dem für die Bewertung eines Objektes die Preise ähnlicher Objekte herangezogen werden, zweitens das Sachwertverfahren, bei dem einem Bodenwert die Herstellungskosten für die baulichen und sonstigen Anlagen zugeschlagen werden und drittens das Ertragswertverfahren, bei dem der Bodenwert über einen mit dem Liegenschaftszins diskontierten Nettomietertrag (Reinertrag) ergänzt wird (Meister und Dressel 2017, S. 138 ff). Letztlich möchte man mit diesen

\footnotetext{
2 Der Beitrag ergänzt mit seiner Monte-Carlo-Simulation im Rahmen eines integrierten Financial Models bayesianische und Fuzzy-Logic-Ansätze zur Erfassung der Unsicherheit bei der Bestimmung des Verkehrswerts (siehe hierzu unter anderem Alkhatib et al. 2015).
} 
Methoden auf den Verkehrswert, also den wahrscheinlichen Transaktionspreis einer Immobilie schließen. Bei diesen „Bewertungsverfahren“ handelt es sich also eher um eine Preisschätzung als um eine Abbildung des intrinsischen, risikogewichteten Wertes.

In einem unvollkommenen Markt können realisierte Marktpreise jedoch von dem nachhaltigen Wert aller möglichen und risikogewichteten Ertragsüberschüsse abweichen. Bei der Ermittlung des Ertragswertes wird (zumindest in Deutschland) der Wert des Bodens und der Wert der Erträge aus der Vermietung der Gebäude(n) separat ermittelt und anschließend summiert. Hierzu wird der Gedanke zugrunde gelegt, dass einerseits die (wirtschaftliche) Nutzungsdauer dieser beiden Komponenten unterschiedlich ist und anderseits der Risikogehalt unterschiedlich sein kann. ${ }^{3}$ Bei der Ermittlung des Bodenwertes wird auf Vergleichspreise zurückgegriffen. Bei der Bestimmung des Gebäudewertes wird der (zukünftige) Reinertrag ${ }^{4}$ über die wirtschaftliche Nutzungsdauer kapitalisiert. Dabei entspricht der Kapitalisierungszinssatz in der Praxis dem Liegenschaftszinssatz. Hierbei wird unterstellt, dass der Liegenschaftszinssatz das Risiko kennzeichnet, dem die zukünftigen Zahlungsflüsse aus der Vermietung unterliegen. Damit ist die Wertermittlung der Gebäude innerhalb des Ertragswertverfahrens ein DCF-Verfahren (Discounted-Cashflow-Verfahren), wobei sich der Liegenschaftszins rückblickend aus früheren Markttransaktionen ableitet. Der Risikogehalt wird folglich aus anderen Transaktionen deduziert.

\section{Grundidee einer risikogerechten Bewertung}

Unter der Annahme, dass auf unvollkommenen Märkten die erzielten Marktpreise von einem risikogerechten, fundamentalen Ertragswert abweichen können, ist es möglich, dass für eine Immobilie gemessen an ihren (unsicheren) zukünftigen Cashflows zu hohe Preise bezahlt werden. Dies führt auch zu einem zu niedrigen Liegenschaftszins in Folgetransaktionen.

Ein weiterer wichtiger Punkt ist, dass bei der Ermittlung des Wertes eines Immobilienportfolios mit Hilfe des Ertragswertverfahrens mit festgesetztem Liegenschaftszinssatz die voneinander unabhängig ermittelten Werte der einzelnen Objekte summiert werden und keine Diversifikationseffekte betrachtet werden; bei größeren Portfolios werden häufig Preisabschläge ermittelt. Weil bei der (risikoadjustierten) fundamentalen Bewertung eines Immobilienportfolios die summierten (unsicheren) Zahlungsüberschüsse des Portfolios bewertet werden, lassen sich Risikodiversifika-

\footnotetext{
3 Der unterschiedliche Risikogehalt wird beim Ertragswertverfahren nicht weiter beachtet. Sowohl bei der Ermittlung des Bodenwerts als auch bei der Ermittlung des Gebäudewerts wird der Liegenschaftszins als (risikogerechter) Diskontierungsfaktor angesehen.

4 Dies ist ein um die Bodenwertverzinsung geminderter Wert der Erträge aus der Vermietung. Dabei wird die Bodenwertverzinsung (in der Praxis) aus der Multiplikation des Bodenwertes mit dem Liegenschaftszinssatz ermittelt. Die Verwendung des Liegenschaftszinssatzes (in der Praxis) zeigt sowohl bei der Bodenwertverzinsung als auch bei der Diskontierung der Reinerträge die (implizite) Annahme des gleichen Risikogehaltes dieser beiden separierten Positionen.
} 
tionseffekte innerhalb des Portfolios berücksichtigen ${ }^{5}$; der Wert einzelner Objekte hängt von den Zahlungen (bzw. von der Risikostruktur) der anderen Objekte ab (siehe hierzu unter anderem Szumilo et al. 2016).

Ergänzend zur Schätzung möglicher Preise (über ein Verkehrswertgutachten) ist folglich eine Berechnung des risikogerechten, fundamentalen Ertragswerts insbesondere bei Immobilienportfolios sinnvoll. Dieser Wert basiert auf den erwarteten zukünftigen Cashflows, die risikogerecht diskontiert werden. Anstelle des Liegenschaftszinses tritt bei den hier vorgeschlagenen DCF-Verfahren ein ,risikogerechter Diskontierungszinssatz" (Kapitalkostensatz).

Die größte Limitierung des üblichen DCF-Verfahrens besteht darin, dass der Diskontierungszinssatz über das Capital Asset Pricing Model geschätzt wird. Dies wäre angemessen, wenn die Kapitalmärkte vollkommen wären und gleichzeitig eine gute Näherung für das Geschehen auf Immobilienmärkten böten. Jedoch sind Immobilienmärkte durch zahlreiche Unvollkommenheiten gekennzeichnet (u. a. sind die Objekte heterogen, Informationen sind asymmetrisch verteilt, es gibt Marktzugangsbarrieren und hohe Transaktionskosten). Bewertungen, die auf der Gültigkeit der Annahmen des CAPM aufbauen, führen dann nicht zu verlässlichen Bewertungen. Diskontierungszinssätze und Renditen werden dann fehlerhaft geschätzt. ${ }^{6}$

Für eine risikogerechte Bewertung einer Immobilie ist es sinnvoll, ausgehend von einer (quantitativen und qualitativen) Risikoanalyse den Wert abzuleiten - ein Rückgriff auf oft nicht vorhandene und zudem für die Zukunft wenig repräsentative Kapitalmarktdaten ist nicht erforderlich. Als Weiterentwicklung des traditionellen (einwertigen) DCF-Verfahrens kommen simulationsbasierte (stochastische) DCFVerfahren zum Einsatz - diese gewährleisten Transparenz über Einzelrisiken und den aggregierten Risikoumfang.

Abb. 1 zeigt den Erwartungswert und eine realistische Bandbreite der bewertungsrelevanten freien Cashflows einer Immobilie, deren Berechnung ausgehend von einer Risikosimulation später im Detail erläutert wird.

Die Grundidee der Bewertung mit dem simulationsbasierten DCF-Verfahren beruht auf der Grundüberlegung, dass ein höherer Umfang an Risiken potenziell zu höheren Planabweichungen führt, was mehr Eigenkapital (Risikotragfähigkeit) erfordert. Und ein zunehmender Bedarf an Eigenkapital führt zu höheren Kapitalkosten (Diskontierungszinssätzen) und somit zu einem niedrigeren fundamentalen Wert.

Notwendig für die Bestimmung eines fundamentalen Wertes ist die Erweiterung des Cashflow-Modells durch die Erfassung von Risiken. Dies kann in der einfachsten Ausgestaltung einer Dreiecksverteilung durch die Angabe von (a) Mindestwert, (b) wahrscheinlichstem Wert und (c) Maximalwert einer Planungsposition (z. B. Mietwachstumsrate oder Leerstandsquote) erfolgen. Ausgehend vom Cashflow-Modell und den geschätzten Risiken kann mit Hilfe von Simulationen eine repräsen-

\footnotetext{
5 Es erfolgt zuerst die Summierung (der Zahlungsüberschüsse) und „,am Ende“ wird bewertet. Die Reihenfolge ist im Vergleich zur Portfoliowertermittlung im Falle des Ertragswertverfahrens umgekehrt.

6 Die Anwendung eines Modells, dessen Annahmen (ganz oder teilweise) verletzt werden, kann nur zufällig richtige Ergebnisse liefern. Allein durch die vorhandene Informationsasymmetrie und vorhandene Fungibilität kann der Immobilienmarkt nicht vollkommen sein und somit kann die Anwendung von CAPM nicht zu dauerhaft richtigen Ergebnissen führen (siehe z. B. Artmann et al. 2012; Hagemeister und Kempf 2010; Hanauer et al. 2013; Chen et al. 2011 oder Walkshäusl 2013).
} 
Abb. 1 Exemplarische Bandbreitengrafik „Free Cashflow“. (Quelle: eigene Darstellung)

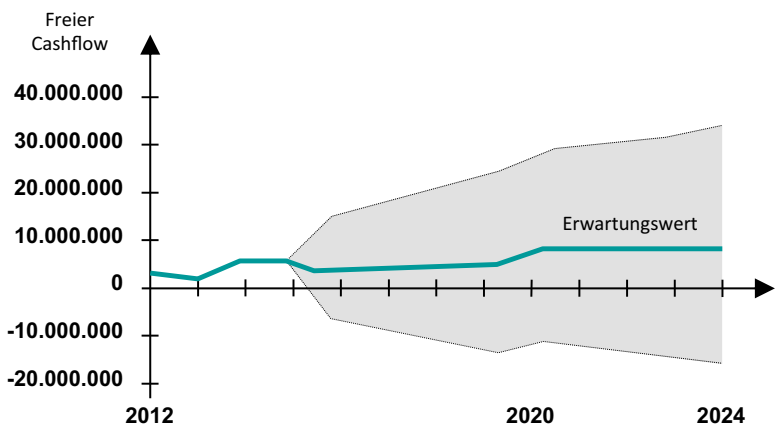

tative Anzahl möglicher Szenarien der Ertrags- und Cashflow-Entwicklung eines Immobilienobjekts bestimmt werden (in Abb. 1 sind die Szenarien aller Entwicklungen innerhalb des markierten Möglichkeitenraums dargestellt). Die Einzelrisiken werden mit Hilfe von Monte-Carlo-Simulationen aggregiert (vgl. hierzu u. a. Gleißner 2017).

Bei der Bestimmung eines fundamentalen Wertes mit dieser Methode ist es nicht relevant, aus welcher wirtschaftlichen Tätigkeit die Cashflows resultieren. Das Vorgehen bei der Bewertung der Cashflows aus einer Immobilie, einem Immobilienportfolio oder aus einem (von Immobilien unabhängigen) Unternehmen ist dem Grunde nach identisch. Ein so gewonnener risikogerechter Wert lässt sich dann mit einem möglichen (z. B. durch ein gutachterliches Verfahren geschätzten) Verkaufspreis vergleichen, um eine etwaige Fehlbewertung einer Immobilie bei aktuellen Marktverhältnissen zu schätzen. Das fundamentale Verfahren zielt auf den hypothetischen Wert, das gutachterliche Verfahren auf den am Markt erzielbaren Preis. Weichen beide Werte stark voneinander ab, könnte dies auf eine Fehlbewertung (auf dem Markt) hindeuten. Vor allem aber liefert die simulationsbasierte Bewertung eine Fülle zusätzlicher Informationen: Für die Risikosteuerung kann es einen Unterschied machen, ob sich der Mittelwert aus allen Szenarien aus sehr eng liegenden Szenarien oder aus sehr weit auseinander liegenden Szenarien folgern lässt. Auch lassen sich Schwankungs- und Insolvenzrisiken modellendogen abbilden sowie die Wahrscheinlichkeit für das Erreichen einer Mindestrendite. Dies ist für die Kalkulation angemessener Fremdkapitalzinsen entscheidend. ${ }^{7}$ Diese zusätzlichen Informationen stehen bei einer Bestimmung der Ertragswerte nach der Immobilienwertermittlungsverordnung (ImmoWertV) nicht zur Verfügung.

\subsection{Simulation zukünftiger Unsicherheiten und Einbettung in Literatur}

Mit Hilfe der Risikosimulationsverfahren (Risikoaggregation mit der Monte-CarloSimulation) sind Rückschlüsse auf das Gesamtrisiko einer Investition, die Pla-

\footnotetext{
7 Eine derartige modellendogene Ableitung der bewertungsrelevanten Ausfallwahrscheinlichkeit, die neben Fremdkapitalzinsen auch die (erwartete) Lebensdauer des Unternehmens bestimmt, ist bei den in ihrer Struktur deterministischen Bewertungsansätzen „Ertragswert als risikoloser Barwert künftiger Sicherheitsäquivalente“ und „Ertragswert als risikoadjustierter Barwert künftiger Erwartungswerte“ nicht möglich.
} 
nungssicherung und die realistische Bandbreite der Cashflows möglich. Dies könnte beispielsweise den freien Cashflow (Zielgröße) einer Investition betreffen. Aus der Vielzahl der ermittelten Realisationen der Zielgröße folgen aggregierte Häufigkeitsverteilungen. ${ }^{8}$ Ausgehend davon kann auf die Risikomaße wie die Standardabweichung der Cashflows oder den Eigenkapitalbedarf (Risk Adjusted Capital oder Risikokapital, RAC) ${ }^{9}$, geschlossen werden.

Risikoaggregationsmodelle gehen immer zunächst von der Planung bzw. Wirtschaftlichkeitsrechnung aus (oder von einer Prognose, falls man als externer Analyst eine Bewertung zu machen hat), die mit den quantifizierten Risiken verknüpft werden.

Hertz (1968) hat in seiner wegbereitenden Arbeit gezeigt, wie Zufallsziehungen aus einer Cashflow-Verteilung bei der Bewertung eines Projekts - und damit auch einer Projektentwicklung - hilfreich sein können. Pyhrr (1973) übertrug dies auf den Fall einer Immobilieninvestition, Wofford (1978) sowie Hughes (1995) übertrugen dies auf Immobilienbewertungen. Bei ihren Vorgehen stand vor allem das Abbilden stochastischer Prozesse bei den Mieten im Fokus. Bis zum Monte-Carlo-Ansatz von Jaffe (1980) beruhten die Modelle auf rein numerischen Lösungen.

Unser Modell fußt auf Modellen von French und Gabrielli (2005); Hoesli et al. (2006) sowie Baroni et al. (2007). Bei Hoesli et al. (2006) wird der Diskontierungszinssatz aus der Simulation abgeleitet. Baroni et al. (2007) sowie Amédée-Manesme et al. (2013) erweitern das Vorgehen um Besonderheiten innerhalb der Portfoliobewertung. Bei ihnen spielt die Modellierung der unsicheren Leerstandsentwicklung eine besondere Rolle. Bei all diesen Arbeiten gehen simulierte Modellprognosen in die Simulation ein. Follain und Giertz (2011) verwenden die durch Simulationsmodelle gewonnenen Ergebnisse für Stresstests. Pfnür und Armonat (2013) legen in ihren Simulationen den Akzent weniger auf die Einnahmen respektive die Preisseite, sie simulieren die Einflüsse unsicherer operativer Kostenbestimmungsfaktoren. Gemeinsam ist allen Ansätzen das Reduzieren von Unsicherheit durch die simultane Berücksichtigung mehrerer möglicher Zukunftsszenarien.

Die in der Bewertungspraxis oft verwendete Sensitivitätsanalyse kann bei einer Bewertung mit Hilfe einer Simulation entfallen, da die vorab bekannten Risiken bereits modellendogen sind. Sensitivitätsanalysen sind sehr sinnvoll, wenn bei einer Bewertung keine Unsicherheit der Parameterwahl zugelassen ist. Dies ist bei der Simulation aber per Definition vorgesehen. Die Bewertung berücksichtigt bei simulationsbasierten Verfahren diese Unsicherheit explizit und wertet die Wirkung der Unsicherheiten konsistent aus.

\footnotetext{
8 Im Unterschied zur Kapitalmarkttheorie für vollkommene Märkte (z. B. Capital-Asset-Pricing-Modell) sind hier systematische und nicht-diversifizierte unsystematische Risiken relevant, was z. B. durch Konkurskosten zu begründen ist.

9 Der Eigenkapitalbedarf (für ein Ziel-Rating) ist der Umfang von Verlusten, der mit einer vom ZielRating abhängigen Wahrscheinlichkeit $\left(\mathrm{p}^{*}\right)$ nicht überschritten wird (und entspricht damit einem Value-atRisk).
} 
Tab. 1 Entscheidungen zur Art der Risikoerfassung. (Quelle: Gleißner 2013)

\begin{tabular}{|c|c|c|}
\hline \multicolumn{3}{|c|}{ Grundsatzentscheidungen zur Risikoerfassung bei der Bewertung } \\
\hline \multirow[t]{2}{*}{$\begin{array}{l}\text { Risikobezugs- } \\
\text { einheit }\end{array}$} & $\begin{array}{l}\text { Ergebnis (Cashflow, } \\
\text { Ertrag) }\end{array}$ & $\begin{array}{l}\text { Insbesondere Bewertung nicht börsennotierter Unter- } \\
\text { nehmen und Immobilien }\end{array}$ \\
\hline & $\begin{array}{l}\text { Relative Preis- } \\
\text { schwankungen (Akti- } \\
\text { enrenditen) }\end{array}$ & $\begin{array}{l}\text { Wenn (a) keine überlegenen Informationen zum Ergeb- } \\
\text { nis vorliegen oder (b) die Bewertung der Aktien eines } \\
\text { börsennotierten Unternehmens erfolgen soll (kurzfris- } \\
\text { tig engagierter Aktionär trägt Aktienkursrisiken) }\end{array}$ \\
\hline \multirow[t]{3}{*}{ Risikomaß } & Standardabweichung & Lageunabhängig, zweiseitig, für handelbare Assets \\
\hline & Value-at-Risk $(\mathrm{VaR})^{\mathrm{a}}$ & $\begin{array}{l}\text { Lageabhängig, Downside-Maß, für nicht-handelbare } \\
\text { Assets (Risiko als möglicher „Verlust“) }\end{array}$ \\
\hline & $\begin{array}{l}\text { Deviation Value-at- } \\
\text { Risk }(\mathrm{DVaR})^{\mathrm{b}}\end{array}$ & $\begin{array}{l}\text { Lageunabhängig, Downside-Maß, für handelbare As- } \\
\text { sets (mögliche „Planabweichung“) }\end{array}$ \\
\hline \multirow[t]{2}{*}{$\begin{array}{l}\text { Berechnungs- } \\
\text { zeitraum }\end{array}$} & $\begin{array}{l}\text { Historische (objekti- } \\
\text { vierbare) Daten }\end{array}$ & $\begin{array}{l}\text { Historische Aktienrenditen oder historische Cashflows } \\
\text { oder Erträge }\end{array}$ \\
\hline & $\begin{array}{l}\text { Zukunftsorientiert } \\
\text { (planungskonsistent) }\end{array}$ & $\begin{array}{l}\text { Szenariobasierte Verfahren oder stochastische Simula- } \\
\text { tionsverfahren }\end{array}$ \\
\hline \multirow{4}{*}{$\begin{array}{l}\text { Risikodiversifikation } \\
\text { beim Bewertungs- } \\
\text { subjekt }(d)\end{array}$} & Perfekt diversifiziert & Nur systematische Risiken ${ }^{\mathrm{c}}$ \\
\hline & $\begin{array}{l}\text { Individueller Risiko- } \\
\text { diversifikationsfaktor }\end{array}$ & $\begin{array}{l}\mathrm{d} \text { hängt vom verfügbaren Vermögen und der Korrelati- } \\
\text { onsstruktur zwischen Marktportfolio, Ergebnissen und } \\
\text { sonstigen Vermögensgegenständen } \mathrm{ab}^{\mathrm{d}}\end{array}$ \\
\hline & Nicht diversifiziert & $\begin{array}{l}\text { Sämtliche Risiken werden getragen, Risikodiversifikati- } \\
\text { onsfaktor } d=1\end{array}$ \\
\hline & $\begin{array}{l}\text { „Mittelmäßig“" diver- } \\
\text { sifiziert }\end{array}$ & $\begin{array}{l}\text { Nicht diversifizierbare und Teile der prinzipiell diversi- } \\
\text { fizierbaren Risiken werden getragen, z. B. „Heuristik“ } \\
d=\frac{1}{2}(1+p) \text { oder } d=0,5\end{array}$ \\
\hline \multirow{5}{*}{$\begin{array}{l}\text { Risikoaggregations- } \\
\text { reihenfolge }\end{array}$} & Risikoprofilmethode & Nur einmalige Risikoadjustierung in Periode $t=1$ \\
\hline & $\begin{array}{l}\text { Endwertverteilungs- } \\
\text { methode }\end{array}$ & $\begin{array}{l}\text { Nur einmalige Risikoadjustierung in Periode } \mathrm{t}=\mathrm{T} \\
\text { Umsetzung eines vollständigen Finanzplans }\end{array}$ \\
\hline & $\begin{array}{l}\text { Periodensicherheits- } \\
\text { äquivalent }\end{array}$ & $\begin{array}{l}\text { Risikoabschlag aus Replikationsmodellen oder Si- } \\
\text { cherheitsäquivalent unmittelbar aus Nutzenfunktion } \\
\text { unabhängig je Periode (vgl. Buch und Dorfleitner } 2007 \\
\text { sowie Laux und Schabel 2009) }\end{array}$ \\
\hline & $\begin{array}{l}\text { Rekursive Sicher- } \\
\text { heitsäquivalente }\end{array}$ & $\begin{array}{l}\text { Sicherheitsäquivalente Bewertungen für die Zahlun- } \\
\text { gen jeder Periode unter Berücksichtigung des Sicher- } \\
\text { heitsäquivalents der Folgeperiode rekursiv bestimmt, } \\
\text { theoretisch optimales Verfahren }\end{array}$ \\
\hline & Zeitlich-horizontal & $\begin{array}{l}\text { Traditionelle Berechnung mit risikoadjustierten Dis- } \\
\text { kontierungszinssätzen (Risikozuschlagverfahren) }\end{array}$ \\
\hline
\end{tabular}

${ }^{a}$ Der Value-at-Risk (zum Wahrscheinlichkeitsniveau $p$ ) drückt aus, welcher Umfang an Verlusten in der Betrachtungsperiode mit einer Wahrscheinlichkeit von $1-p$ nicht überschritten wird und ist damit als ,Eigenkapitalbedarf" interpretierbar, der prinzipiell nicht diversifizierbar ist (damit entspricht der sogenannte Risikodiversifikationsgrad $d$ der Korrelation der Cashflows gegenüber dem Marktportfolio $(\varrho)$ )

${ }^{b}$ Der Deviation Value-at-Risk zu einem vorgegebenen Wahrscheinlichkeitsniveau $p$ drückt aus, welcher Umfang einer negativen Planabweichung (gegenüber dem Erwartungswert) in der Betrachtungsperiode mit einer Wahrscheinlichkeit von $1-p$ nicht überschritten wird

${ }^{c} \varrho$ : Korrelation zwischen Bewertungssubjekt und Marktportfolio. Risikodiversifikationsfaktor $d=\varrho$

${ }^{\mathrm{d}}$ Vgl. Gleißner und Wolfrum (2008) zur Herleitung der Bewertungsgleichungen 


\subsection{Bestimmung des risikogerechten Wertes}

Die Bestimmung des risikogerechten Ertragswerts basiert auf dem DCF- oder dem Ertragswertverfahren. Simulationsbasierte Verfahren ergänzen diese Modelle durch die Abschätzung von Wahrscheinlichkeiten für die relevanten fundamentalen Werttreiber. Für eine risikogerechte Bewertung wird dann festgelegt, in welcher Form Risiken im DCF-Verfahren erfasst werden.

Dabei sind Entscheidungen zu der Art der Risikoerfassung zu treffen, insbesondere bezüglich:

- Risikobezugseinheit

- Risikomaß

- Berechnungszeitraum (Risikoquantifizierungszeitraum)

- Risikodiversifikation beim Bewertungssubjekt

- Risikoaggregationsreihenfolge.

Tab. 1 erläutert diese Bezugseinheiten anhand möglicher Maße. Die kursiv dargestellten Felder stellen eine empfohlene Vorgehensweise bei der Bewertung von Immobilien dar.

\section{Zur Ermittlung des risikogerechten Werts von Immobilien}

Bei der Immobilienbewertung sollten aufgrund unterschiedlicher Risikoprofile Boden und Gebäude getrennt bewertet werden. Der Ertragswert der Gebäude wird aus den Zahlungen für die Gebäudenutzungen abgeleitet, der Wert des Bodens aus der Pacht, welche durch den Boden erwirtschaftet werden könnte. Diese Aufteilung ist erforderlich, da die Gebäude mit endlicher wirtschaftlicher Lebensdauer betrachtet werden, während der Boden mit unendlicher Lebensdauer bewertet wird. In Abschn. 4.1 werden die methodischen Grundlagen der Bewertung der Gebäude dargestellt während in Abschn. 4.2 der Wert des Bodens angesprochen wird.

\subsection{Wert der Gebäude: Bewertung der laufenden Zahlungen}

Die Gruppe der DCF-Bewertung beinhaltet zwei methodische Untergruppen, die sich in der Risikoaggregationsweise unterscheiden: die Risikozuschlagsmethode zum einen und die Sicherheitsäquivalenzmethode oder Risikoabschlagsmethode zum anderen. Bei den in der Praxis durchgeführten DCF-Bewertungen dominiert die Risikozuschlagsmethode, also das Vorgehen, bei dem die Risiken im Diskontierungssatz (als Zuschlag auf den risikolosen Zinssatz) berücksichtigt werden. Die Sicherheitsäquivalenzmethode vermeidet Probleme der Zuschlagsmethode, stellt aber keinen (risikoadäquaten) Diskontierungszinssatz als Vergleich zum Liegenschaftszins bereit. In diesem Kapitel werden diese beiden Methoden verglichen und eine praxistaugliche Kombination der beiden Methoden dargestellt, mit deren Hilfe die Nachteile der Risikozuschlagsmethode umgegangen werden und ein risikoadäquater Diskontierungszinssatz dargestellt werden kann. 
Als relevante Ergebnisgröße für die Bewertung der Zahlungen $(Z)$ einer Immobilie wird der periodenbezogene freie Cashflow nach Steuer $(F C F)$ nach Abzug der Bodenwertverzinsung betrachtet. ${ }^{10}$

Durch die durchgehende Berücksichtigung von Nach-Steuer-Größen wird der Vorteil durch die steuerliche Abzugsfähigkeit der Fremdkapitalzinsen schon im Zahlungsstrom berücksichtigt und muss nicht explizit behandelt werden. Dabei wird die Bodenwertverzinsung als Produkt des Bodenwertes $\left(B W_{t}\right)$ der Periode und des risikogerechten Bodenwertverzinsungssatz $\left(r_{l}\right)$ gemäß Verkehrswertgutachten bestimmt:

$$
F C F_{t}=F C F \_v o r \_B W v e r z i n s u n g_{t}-B W_{t} * r_{l}
$$

\subsubsection{Risikozuschlagsmethode (Erfassung der Risiken im Nenner):}

Bei der Risikozuschlagsmethode wird ein Zuschlag (Risikozuschlag, $r_{Z}$ ) in Abhängigkeit von der vorhandenen Risikomenge $R\left(\widetilde{Z}^{\prime}\right)$ und in Abhängigkeit von dem auf dem Markt herrschenden Risikopreis $\lambda_{R Z}$ zum risikolosen Zinssatz $\left(r_{f}\right)$ addiert, um so einen Diskontierungszinssatz $k$ (näherungsweise dem Kapitalkostensatz) für die Diskontierung der zukünftig erwarteten Zahlungen $\left(E\left(\widetilde{Z}_{t}\right)\right) \mathrm{zu}$ erhalten $\left(k=r_{f}+r_{z}\right) . R\left(\widetilde{Z}^{\prime}\right)$ ist hierbei der normierte bewertungsrelevante Risikoumfang (in \% des Objektwerts). Es ist also ein „Renditerisikomaß“, welches in Abhängigkeit des Risikoumfangs des Objektes $\left(R^{0}\left(\widetilde{Z}_{1}^{\prime}\right)\right)$ und des Diversifikationsfaktors zum Restvermögen $(d)^{11}$ des Bewertungssubjekts bestimmt werden kann. ${ }^{12}$ Der Diversifikationsfaktor ist eine Größe, die vom Bewertungssubjekt abhängt und erfasst, dass dieser im Kontext seines Gesamtvermögens einen Teil der Risiken des Bewertungsobjekts diversifizieren kann. Das bewertungsrelevante Risiko des Bewertungsobjektes (im Rahmen einer Gesamtvermögensbetrachtung) verändert sich $^{13}$ im Vergleich zu einer Einzelfallbetrachtung des Bewertungsobjektes. ${ }^{14} \mathrm{Zu}$ beachten ist, dass der Diskontierungszinssatz $k$ - und damit der Wert einer Immobilie - auch abhängig vom Rendite-Risiko-Profil verfügbarer Alternativanlagen ist. Dies wird durch die Größe $\lambda_{R Z}$ erfasst, den „Marktpreis des Risikos“. So berechnet sich der risikogerechte, innere Wert des erwarteten Zahlungsstroms $W(\widetilde{Z})$ wie folgt:

\footnotetext{
10 Entsprechend wird der Wert des Bodens auf den Wert der Zahlungen addiert, um den Gesamtwert zu erhalten.

11 Der Diversifikationseffekt mit dem Restvermögen ist nicht mit dem Diversifikationseffekt innerhalb des Bewertungssubjektes zu verwechseln. Letzteres entsteht z. B. durch mehrere Mieteinheiten innerhalb der betrachteten Immobilie. Ersteres durch das Vorhandensein anderer Assets des Bewertungssubjektes (z. B. Unternehmensanteile).

12 Das bewertungsrelevante Risiko des Bewertungsobjekts $R\left(\widetilde{Z}_{1}^{\prime}\right)$ ist damit $R^{0}\left(\widetilde{Z}_{1}^{\prime}\right) \cdot d$, d. h. es ist abhängig vom Risiko des Bewertungsobjekts und den eigenen Diversifikationsmöglichkeiten (d).

13 Im Normalfall verringert sich dieser durch Risikodiversifikation.

14 Die Ermittlung des Diversifikationsfaktors ist je nach Struktur des Gesamtvermögens und je nach gewähltem Risikomaß unterschiedlich. Unter Annahmen des CAPM entspricht der Diversifikationsfaktor der Korrelation mit dem Gesamtmarkt.
} 


$$
\begin{aligned}
W\left(\widetilde{Z}_{1}\right) & =\frac{E\left(\widetilde{Z}_{1}\right)}{1+k}=\frac{E\left(\widetilde{Z}_{1}\right)}{1+r_{f}+r_{z}}=\frac{E\left(\widetilde{Z}_{1}\right)}{1+r_{f}+\lambda_{R Z} \cdot R\left(\widetilde{Z}_{1}^{\prime}\right)} \\
& =\frac{E\left(\widetilde{Z}_{1}\right)}{1+r_{f}+\lambda_{R Z} \cdot R^{0}\left(\widetilde{Z}_{1}^{\prime}\right) \cdot d}
\end{aligned}
$$

Es wird angenommen, dass - ähnlich zum CAPM - jeder Investor zwei leicht verfügbare alternative Investitionen tätigen kann: die Investition in (nahezu) risikolose Anlagen (z. B. Staatsanleihen sehr guter Bonität) einerseits und die Investition in ein (empirisches) ,Marktportfolio“ anderseits. ${ }^{15}$ Letzteres umfasst alle riskanten Investitionsmöglichkeiten. Geht man von diesen Alternativinvestments aus und verwendet die Standardabweichung als Risikomaß, so ist $\lambda_{R Z}$ gerade die Marktrisikoprämie ${ }^{16}$ in Relation zur Standardabweichung der Marktrendite (die sogenannte „Sharpe Ratio“).

Die Risikozuschlagsmethode hat zwei zentrale Nachteile:

- Die Cashflow-Risiken werden nicht adäquat abgebildet: In der Praxis der risikogerechten Bewertung besteht das Fehlerpotenzial darin, dass in Gl. 2 der eigentliche Risikoumfang des Bewertungsobjekts (Immobilien), also z. B. die Standardabweichung der Cashflows, gar nicht unmittelbar einfließt und auch nicht hinreichend approximiert wird. Das Risikomaß bezieht sich auf (im Allgemeinen nicht bekannte) „relative Wertschwankungen“. Allerdings existieren für die meisten Immobilien und Immobilienportfolien keine historischen Renditeschwankungen. Außerdem lässt sich selbst aus historischen Renditeschwankungen nicht auf das bewertungsrelevante zukünftige Risiko einer Immobilie oder eines Immobilien-Portfolios schließen.

- Einheitlicher Zuschlag für positive und negative Zahlungen: Aufgrund der Risikoaversion soll durch die Diskontierung unsicherer Zahlungen ein niedrigerer Wert zugewiesen werden als für sichere Zahlungen. Genau dies wird bei der Diskontierung (möglicherweise) negativer Zahlungsströme, z. B. bei einer zukünftigen Renovierung, nicht erreicht.

\subsubsection{Sicherheitsäquivalentmethode (S̈̈) - Erfassung der Risiken im Zähler}

Aufgrund dieser Mängel der Risikozuschlagsmethode ist die Sicherheitsäquivalentmethode (S⿱艹) sinnvoller, da die genannten Probleme nicht auftreten.

$$
W\left(\widetilde{Z}_{1}\right)=\frac{S \ddot{A}\left(\widetilde{Z}_{1}\right)}{1+r_{f}}=\frac{E\left(\widetilde{Z}_{1}\right)-\lambda_{S \ddot{A}} \cdot R\left(\widetilde{Z}_{1}\right)}{1+r_{f}}=\frac{E\left(\widetilde{Z}_{1}\right)-\lambda_{S \ddot{A}} \cdot R^{0}\left(\widetilde{Z}_{1}\right) \cdot d}{1+r_{f}}
$$

\footnotetext{
15 Dabei wird nicht davon ausgegangen, dass CAPM Gültigkeit hat. Die Annahmen des CAPMs werden nicht übernommen; dies ist auch nicht notwendig. Es wird lediglich für den Beispielfall die gleiche Menge an (relevanten) Alternativinvestment genommen, wie beim CAPM. In explizit betrachteten Einzelfällen sind andere Alternativinvestments möglich.

16 Das ist die Differenz der erwarteten Rendite des Marktportfolios zur risikolosen Anlage.
} 
Der Risikoumfang einer Zahlung wird mit einem Abschlag im Zähler erfasst. Mehr Risiko (z. B. Cashflow-Unsicherheit) führt zu einem niedrigeren Wert. $R(\widetilde{Z})$ ist das Risikomaß und zeigt den bewertungsrelevanten Umfang des Risikos der zu bewertenden Zahlung oder Erträge (in Geldeinheiten). Dies könnte z. B. die Standardabweichung der Cashflows sein und kann - wie schon oben erläutert - auf das Risiko des Objekts und den Diversifikationsfaktor $d$ des Bewertungssubjekts aufgeteilt werden.

Die Risikopräferenz $\left(R\left(Z_{1}\right)\right.$ im Zähler) und Zeitpräferenz ${ }^{17}$ ( $r_{f}$ im Nenner) werden bei dieser Vorgehensweise klar unterschieden (vgl. Ballwieser 1981).

Die Risikoanalyse der zu bewertenden Zahlungen (Cashflows) oder Erträge führt zu planungs- und risikogerechten Risikomaßen, die nicht - wie der Beta-Faktor des CAPM - aus historischen (Aktien-)Renditen abgeleitet werden. Geeignete Risikomaße können z. B. DVaR (siehe auch Tab. 1) oder die Standardabweichung sein.

\subsubsection{Zwischenfazit zur Methodik}

Diese Verfahren für eine risikogerechte Bewertung lassen sich grundsätzlich auf alle Bewertungsobjekte anwenden. Die Einheitlichkeit des Vorgehens einer solchen risikogerechten Bewertung liegt daran, dass hier ein Investor die erwarteten Erträge und die mit ihnen verbundenen Risiken simultan berücksichtigt. Bei gleichem Ertrag und Risiko folgt zu jedem Zeitpunkt auch derselbe ermittelte Wert.

In der Praxis wird bei einer Bewertung meistens der Wert der Zahlungen der einzelnen Perioden ermittelt und anschließend summiert. Bei einer Immobilie mit 50 Jahren Nutzungsdauer $(\mathrm{T}=50)$ (angelehnt an Gl. 3, ohne den Bodenwert) entspräche dies Gl. 4:

$$
W(\widetilde{Z})=\sum_{t=1}^{T=50} \frac{E(\widetilde{Z})}{(1+k)^{t}}
$$

Bei diesem Vorgehen ist es nicht einfach, das Gesamtrisiko über die Laufzeit des Projektes zu beziffern. Durch eine Veränderung der Aggregationsreihenfolge ist jedoch auch dies möglich.

In der in Gl. 4 dargestellten Vorgehensweise wird von einem konstanten Diskontierungszinssatz ausgegangen. Die Berücksichtigung der Risiken im Nenner unterstellt eine Abhängigkeit der risikobedingten Abweichungen der Periode von den Risiken der Vorperiode.

\subsubsection{Ergänzung der Methodik: die Risikoprofilmethode}

In den bisher dargestellten Methoden erfolgte zuerst die Bewertung der einzelnen Perioden aus heutiger Sicht und anschließend wurden diese Werte summiert. Bei der Risikoprofilmethode, die im folgenden Beispiel verwendet wird, dreht sich die Herangehensweise. Zuerst werden die möglichen Ergebnisse aufaddiert und an-

17 Bamberg et al. (2006) sehen im risikolosen Zinssatz präziser „nur“ einen Parameter des Handlungsfelds, der nicht unbedingt der Zeitpräferenz entspricht. 
Abb. 2 Aggregation der Daten nach der stochastisch-dynamischen Ertragswertberechnung. (Quelle: Ballwieser und Hachmeister 2016)

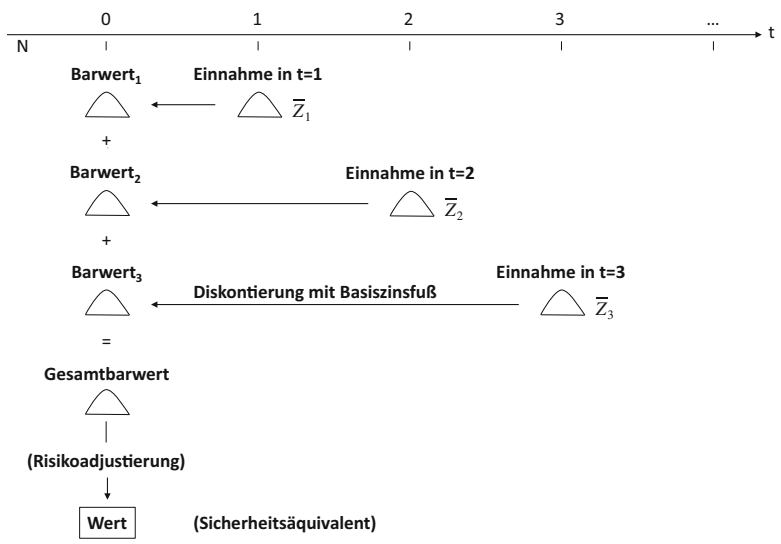

schließend erfolgt die Bewertung dieser Summe. Es wird also zunächst mit Hilfe der risikolosen Diskontierung eine Verteilung der risikobehafteten summierten, bewertungsrelevanten Einzahlungsüberschüsse aus heutiger Sicht erzeugt (siehe Abb. 2). ${ }^{18}$

Die so erzeugte Verteilung kann gemäß den oben skizzierten Methoden bewertet werden, also gemäß Gl. 3 als:

$$
\begin{aligned}
W(\widetilde{Z}) & =S \ddot{A}\left(\sum_{t=1}^{T} \frac{\widetilde{Z}_{t}}{\left(1+r_{f}\right)^{t}}\right) \\
& =E\left(\sum_{t=1}^{T} \frac{\widetilde{Z}_{t}}{\left(1+r_{f}\right)^{t}}\right)-\lambda_{S \ddot{A}, T} \cdot R^{\prime}\left(\sum_{t=1}^{T} \frac{\widetilde{Z}_{t}}{\left(1+r_{f}\right)^{t}}\right) \cdot d
\end{aligned}
$$

Anstelle periodenbezogener Zahlungen tritt hier die Summe der unsicheren Barwerte der einzelnen Perioden. In der Gleichung tritt der risikolose Zinssatz $r_{f}$ wieder auf, der den Vergleich von Zahlungen zu unterschiedlichen Zeitpunkten ermöglicht. Dabei ist zu erwähnen, dass durch diese Betrachtung ein zeitlicher Risikodiversifikationseffekt auftritt. ${ }^{19} \mathrm{Zu}$ beachten ist, dass der das Rendite-Risiko-Profil der Alternativanlagen charakterisierende „Marktpreis des Risikos“ “ $\lambda_{S \ddot{A}}$ nun den Zeitindex $T$ trägt $\left(\lambda_{S \ddot{A}, T}\right)$. Hier ist nämlich zu berücksichtigen, dass das Rendite-Risiko-

\footnotetext{
${ }^{18}$ Eine methodisch verwandte Variante der Bewertung ist die Methode der Endwertverteilung, wo die Verteilung am Ende des Betrachtungszeitraums gebildet wird. Vgl. Ballwieser und Hachmeister (2016).

19 Dieser Effekt ist vergleichbar mit dem Risikodiversifikationseffekt von mehreren Projekten. Die Verluste einer Periode können durch die Gewinne der Folgeperiode (unter Berücksichtigung einer passenden risikolosen Diskontierung) teilweise oder vollständig ausgeglichen werden, wie die Verluste eines Projektes mit den Gewinnen von anderen (parallelen) Projekten ausgeglichen werden können. Im einfachsten Fall - bei voneinander unabhängigen Perioden/Projekten mit jeweilig gleicher Standardabweichung - beträgt damit die Standardabweichung der Gesamtverteilung $\sigma \cdot \sqrt{n}$, Im Vergleich dazu folgt bei der Betrachtung der Schwankung der einzelnen Perioden als das relevante Risiko ein Gesamtrisiko von $\sigma \cdot n$.
} 
Profil der Alternativanlagen (Marktportfolio und risikolose Anlage) nicht nur über eine Periode, sondern über den gesamten Planungszeitraum $(T)$ zu berechnen ist: ${ }^{20}$

$$
\lambda_{T}=\frac{E\left(\prod_{t=1}^{\mathrm{T}}\left(1+\widetilde{r_{m}}\right)-\left(1+r_{f}\right)^{T}\right)}{R\left(\prod_{t=1}^{\mathrm{T}}\left(1+\widetilde{r_{m}}\right)\right)}
$$

Im Zähler steht das erwartete Endvermögen einer Investition in das Marktportfolio $E\left(\prod_{t=1}^{\mathrm{T}}\left(1+\widetilde{r_{m}}\right)\right)$ in Relation zum Endvermögen einer Investition in eine risikolose Anlage $\left(1+r_{f}\right)^{T}$. Im Nenner steht der Risikoumfang der Anlage in das Marktportfolio im Gesamtzeitraum von $T$ Perioden $R\left(\prod_{t=1}^{T}\left(1+\widetilde{r_{m}}\right)\right)$.

Die Berechnung kann auch mit Hilfe der Risikozuschlagsmethode durchgeführt werden, um äquivalente Werte zu erhalten. Dadurch ist auch die Berechnung des Wertäquivalents des risikoadjustierten Diskontierungssatzes $k$ möglich.

Ein Vorteil dieser Bewertungsvorgehen ist, dass dadurch das Beteiligungsrisiko einfach ermittelt werden kann, nämlich als Unterschied zwischen dem risikogerechten Ertragswert aus heutiger Sicht - ermittelt als diskontierter Mittelwert wie in Gl. 5 - und dem ermittelten Quantil des (unsicheren) zukünftigen Ertragswerts.

\subsection{Berücksichtigung des Bodenwertes}

Der Wert des Bodens entsteht aus den (theoretischen) Pachtzahlungen. ${ }^{21}$ Wurden diese Zahlungen beim Cashflow berücksichtigt, muss anschließend der Bodenwert auf den Ertragswert der Cashflows aus den Gebäuden addiert werden, um den Wert des Gesamtimmobilienportfolios zu bestimmen.

Weiterhin muss vorhandenes, nicht betriebsnotwendiges Sondervermögen berücksichtigt werden. Somit ergibt sich der Wert für die Immobilie, W(Immo), als Summe aus dem Wert der liquiden Mittel aus der Immobiliennutzung, W(LiMi), dem Wert des Bodens, W(Boden) und dem Wert der Sondervermögen, W(Sondervermögen).

\footnotetext{
20 Die hier dargestellte Berechnungsformel geht von einer periodenunabhängigen Normalverteilung der Marktrendite aus und rechnet die Rendite über die Laufzeit aus den Renditen der Zwischenperioden aus. Die allgemeine Formulierung (ohne Annahmen über die Verteilung) ist, dass die (mehrperiodige) Differenz von Markt- und risikoloser Rendite über die Gesamtlaufzeit T durch das Risiko der Marktrendite über die Gesamtlaufzeit T dividiert wird.

21 Alternativ kann der Bodenwert aus Vergleichsdaten abgeleitet werden wie aus einem Verkehrswertgutachten.
} 


\section{Anwendung auf einen leicht verfremdeten, realen Fall}

\subsection{Motivation des Vorgehens}

Die theoretischen Überlegungen werden im Folgenden anhand eines Beispiels veranschaulicht. Hierfür wird das Wohnimmobilien-Portfolio des hypothetischen Unternehmens „Immo AG“, eines Bestandshalters von Wohnimmobilien, bewertet. Ergänzend zu den vorliegenden Verkehrswertgutachten möchte der Vorstand den risikogerechten Ertragswert seines Portfolios aufgezeigt bekommen, und zwar unter Einrechnung von Diversifikationseffekten innerhalb des Portfolios.

Auf diesem Weg erwartet der Vorstand folgende Vorteile:

1. Er möchte eine Preisuntergrenze ermitteln, unterhalb derer ein Verkauf des Immobilien-Portfolios keinen Mehrwert bringt.

2. Er möchte durch den nachvollziehbaren Beleg des Wertbeitrags einer geeigneten Risikodiversifikation auf Portfolioebene zeigen, dass sein Portfolio wertvoller als die Summe der Verkehrswerte ist.

Die folgende Bewertung der Immo AG basiert auf den unsicheren freien Cashflows nach Bodenwertverzinsung mit Hilfe der Risikoprofilmethode mit Sicherheitsäquivalent. Hierzu werden die zukünftigen unsicheren Cashflows mit einer Simulation errechnet und anschließend entsprechend den dargestellten Verfahren verdichtet. Durch die Anwendung der erläuterten Methoden war ein Rückgriff auf historische Aktienrenditen wie beim Beta-Faktor des CAPM nicht erforderlich.

Zudem wird im Folgenden die oft vernachlässigte Wirkung der Insolvenzwahrscheinlichkeit $(p)$ berücksichtigt. Die Insolvenzwahrscheinlichkeit ergibt sich automatisch aus der Risikosimulation, weil risikobedingt mögliche Szenarien, die zur Illiquidität führen, einen Abbruch der Zahlungen an die Eigentümer zur Folge haben. Die relative Häufigkeit solcher Szenarien führt unmittelbar zur Insolvenzwahrscheinlichkeit. Diese wirkt letztlich wie eine negative Wachstumsrate.

\subsection{Ausgangsparameter des Praxisbeispiels}

Es wird das Wohnimmobilienportfolio der Immo AG mit folgenden Eckdaten betrachtet. Alle Angaben wurden an ein reales Beispiel angelehnt.

- Bodenfläche: $12.000 \mathrm{~m}^{2}$

- Mietfläche: $5400 \mathrm{~m}^{2}$

- Bilanzwert Grundstücke: 2,4 Mio. EUR

- Bilanzwert Gebäude: 4,6 Mio. EUR

- Verkehrswert (gemäß SachV): 7,5 Mio. EUR

- Liegenschaftszinssatz: $5,2 \%$

- Bodenwertverzinsung: $3,2 \%$

- Fremdfinanzierung: 5,0 Mio. EUR (endfällig) 
Tab. 2 Einheiten aus dem Immobilienportfolio. (Quelle: eigene Annahmen)

\begin{tabular}{llll}
\hline & Fläche je Einheit $\left(\mathrm{m}^{2}\right)$ & Anzahl Einheiten & $\begin{array}{l}\text { Marktmiete am } \\
\text { Anfang }\left(\mathrm{EUR} / \mathrm{m}^{2}\right)\end{array}$ \\
\hline Bau 1 - 1 Zimmer Wohnung & 40,0 & 2 & 7 \\
Bau 1 - 3 Zimmer Wohnung & 70,0 & 40 & 6 \\
Bau 1 - 4 Zimmer Wohnung & 110,0 & 20 & 6,5 \\
Bau 1 - 5 Zimmer Wohnung & 140,0 & 10 & 6 \\
Bau 2 - 3 Zimmer Wohnung & 75,0 & 23 & 6 \\
Bau 3 - 4 Zimmer Wohnung & 80,0 & 1 & 7,5 \\
\hline
\end{tabular}

- Anzahl Mieteinheiten:

- Zahl der Wohneinheiten: 96

- Zahl der Stellplätze: 49

- Zahl der Mietergärten: 32

- Vermietungsstand: $95 \%$

Insgesamt wird sowohl die Mikro- als auch Makrolage als günstig eingeschätzt und die qualifizierte Analyse des Portfolios plausibilisiert die qualitative Prognose.

Die durchschnittliche erwartete Nutzungsdauer wird auf 60 Jahre geschätzt. Für die nächsten Jahre liegt eine Planung des Managements inklusive Mieteinnahmen und Instandhaltungskosten vor. Es wird erwartet, dass die Qualität und Wettbewerbsfähigkeit mit den geplanten Instandhaltungskosten aufrechterhalten werden kann. Es ist wesentlich, dass - im Gegensatz zu traditionellen Bewertungsgutachten - Unsicherheiten über die zukünftige Entwicklung z. B. von Mieten und Kosten im Rahmen der risikogerechten Bewertung explizit berücksichtigt werden. Scheingenauigkeiten in Form von quasi-deterministischen Annahmen werden so vermieden.

Die Planung dient damit als Aufhänger für die Berücksichtigung von Risikowirkungen. Durch Risikoanalysen werden Bandbreiten der zukünftigen Entwicklung anstelle von Punktschätzungen hergeleitet. Somit können Entwicklungskorridore für die Werte dargestellt und analysiert werden.

In dem betrachteten Immobilienportfolio sind die in Tab. 2 dargestellten Einheiten vorhanden.

\subsection{Annahmen für die Modellierung der Risiken ${ }^{22}$}

Um den risikogerechten Objektwert zu ermitteln, werden die Risiken von allen relevanten Modellparametern erfasst und in einer Simulation aggregiert. Die Planungen werden inklusive Unsicherheit für die Laufzeit der Nutzungsdauer fortgeschrieben. Damit erhält man für die gesamte Laufzeit aus der Simulation Bandbreiten für die Cashflows. Die Annahmen basieren auf Plausibilitätsüberlegungen, sie sind damit letztlich subjektive Einschätzungen. Wo es sinnvoll erscheint, wurden Referenzwerte aus der Vergangenheit für einzelne Parameter verwendet. Solche historischen Referenzwerte enthalten ebenfalls ein subjektives Werturteil, nämlich jenes, dass die

${ }^{22}$ Die Annahmen wurden durch die Autoren aufgestellt, angelehnt an ein durchgeführtes Projekt. 
Parameterwelt von morgen hinreichend gut durch die Parameterverteilung in der Vergangenheit approximiert werden kann. Die folgenden Modellparameter fanden mit den angegebenen Wahrscheinlichkeiten Eingang in die Berechnungen:

\subsubsection{Belegung der Mieteinheiten}

- Verlängerungswahrscheinlichkeit der Mietverträge: $93 \%$

- Ausfallwahrscheinlichkeit der Mieter: $2 \%$

- Leerstandsdauer zwischen Mietern (in Monaten): Dreiecksverteilt; Min: 1; wahrscheinlich: 3; Max: 4

Um die unbegrenzte Vertragslaufzeit und das vorhandene Kündigungsrecht der Mieter zu modellieren, wurden eine fiktive Vertragslaufzeit von drei Monaten und die obigen Parameter zu Wahrscheinlichkeit und Wirkung einer Beendigung von Mietverhältnissen unterstellt. In einer erweiterten Modellierung könnte man auch die Unsicherheit solcher Modellparameter auswerten, d.h. man könnte z. B. angeben, dass die maximale Leerstandsdauer selbst unsicher ist (und die Schätzung zwischen 4 und 10 Monaten liegt).

\subsubsection{Einnahmen}

- Inflation (u. a. zur Inflationierung von Verträgen): Stochastischer Mean-Reversion Prozess. $^{23}$

Es wurde eine $80 \%$ ige Inflationierung der laufenden Verträge unterstellt: dabei wird die Inflation als unsicherer Prozess modelliert.

- (inflationsbereinigte) Entwicklung des Marktpreises: Stochastischer Mean-Reversion Prozess mit Zyklus ${ }^{24}$

Abb. 3 Bandbreite der möglichen Inflation in \% p. a. (Quelle: Eigene Berechnung)

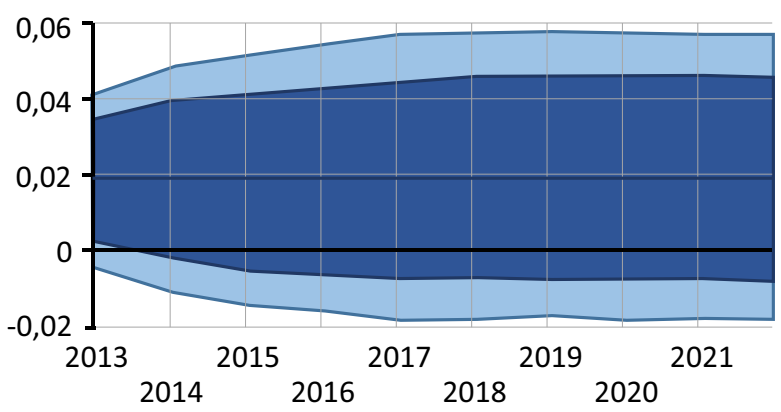

23 Mean-Reversion mit Startwert von $2 \%$, langfristigem Mittelwert von $2 \%$, einer Anpassungsdauer von fünf Jahren und einer Standardabweichung der Schwankungen von 1,0\%. Alle diese Vorgaben lassen sich nach Maßgabe der Einschätzung des Bewerters festlegen.

24 Grundprozess: Mean-Reverting mit Startwert von 2\%, langfristigem Mittelwert von 2,0\%, einer Anpassungsdauer von 5 Jahren und einer Standardabweichung der Schwankungen von $1 \%$ zzgl. eines Zyklus mit 8 Jahren Wellenlänge und 3\%iger maximaler Abweichung vom Grundprozess. Alle diese Vorgaben lassen sich nach Maßgabe der Einschätzung des Bewerters festlegen. 
Abb. 4 Bandbreite des möglichen Marktzyklus; inflationsbereinigte Änderung des Marktpreises gg. Vj. (Quelle: Eigene Berechnung)

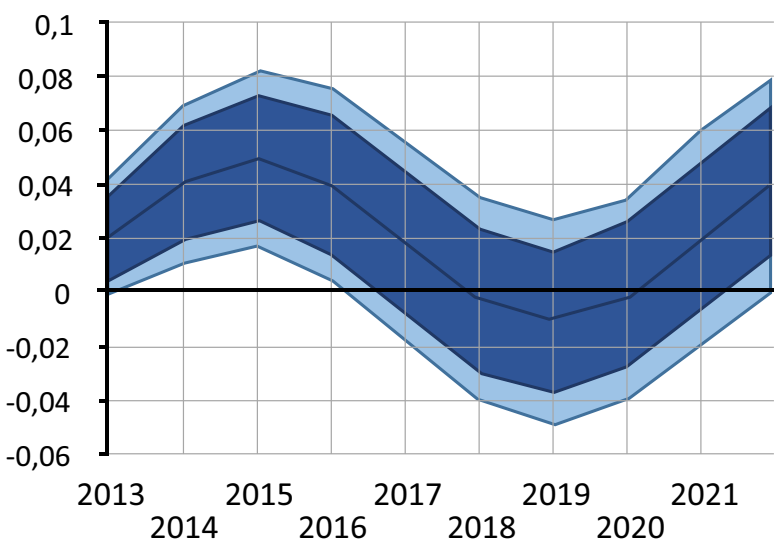

Tab. 3 Annahmen für das bewertete Wohnungsportfolio der Immo AG. (Quelle: Eigene Annahmen)

\begin{tabular}{lllll}
\hline & \multicolumn{4}{l}{ Abweichung der Mieten vom Vergleichsmieten beim Neuvertrag } \\
& $\begin{array}{l}\text { Min } \\
\text { (in \%) }\end{array}$ & $\begin{array}{l}\text { Wahrscheinlich } \\
\text { (in \%) }\end{array}$ & $\begin{array}{l}\text { Max } \\
\text { (in \%) }\end{array}$ & $\begin{array}{l}\text { Erwartungswert } \\
\text { (in \%) }\end{array}$ \\
\hline Bau 1 - 1 Zimmer Wohnung & $-14,29$ & 0,00 & 14,29 & 0,00 \\
Bau 1 - 3 Zimmer Wohnung & $-25,00$ & 0,00 & 33,33 & 2,78 \\
Bau 1 - 4 Zimmer Wohnung & $-23,08$ & 0,00 & 15,38 & $-2,56$ \\
Bau 1 - 5 Zimmer Wohnung & $-16,67$ & 0,00 & 25,00 & 2,78 \\
Bau 2 - 3 Zimmer Wohnung & $-16,67$ & 0,00 & 36,67 & 6,67 \\
Bau 3 - 4 Zimmer Wohnung & $-6,67$ & 0,00 & 6,67 & 0,00 \\
\hline
\end{tabular}

Die Mietentwicklung wurde aufgespalten in ein ausschließlich inflationsbedingtes Wachstum (vgl. Abb. 3) und eine von Mikro- und Makrolage abhängige Entwicklung der Realmiete, die einem idealtypischen Zyklus (vgl. Abb. 4) folgt. Wichtig hierbei ist, dass auch dieser „Zyklus“ keine sichere Beschreibung der Zukunft darstellt, sondern die zukünftige Entwicklung in einer Bandbreite beschreibt.

- Abweichung der Mieten vom auf den Markt üblichen Mieten (Vergleichsmieten) zum Zeitpunkt des Neuvertrags.

Die Risiken in Bezug auf die neu vereinbarten Mieten gegenüber Vergleichsmieten wurden, wie in Tab. 3 dargestellt, erfasst. ${ }^{25}$

\subsubsection{Sonstige unsichere Modellparameter}

- wirtschaftliche Restnutzungsdauer: Gleichverteilt; zwischen 54 und 66 Jahren Zusätzlich wurde auch die Restnutzungsdauer der Gebäude als nicht sicher bekannt modelliert.

\footnotetext{
${ }^{25}$ Schon durch dieses einfache, an die Szenariotechnik angelehnte Verfahren, ist der Bewerter in der Lage, Planungsunsicherheiten genau abzubilden, die bei Eingabe lediglich eines einzigen Planwerts nicht erkennbar sind.
} 


\subsubsection{Erweiterungsmöglichkeiten}

Es gibt viele Möglichkeiten, die Modellierung der Risiken zu verfeinern (z. B. durch Verwenden einer Beta-Verteilung, stochastischer Prozesse, Einführung von ereignisorientierten Risiken, etc.). Um einen Marktpreiszyklus zu vermeiden, ließe sich die Zyklusannahme mit einer Verteilungsfunktion unterlegen. Es könnte z. B. formuliert werden, dass solch ein Zyklus nur mit einer Wahrscheinlichkeit von $40 \%$ vorliegt. Doch diese Erweiterungen könnten aufgrund der höheren Komplexität die Umsetzung in der Praxis gefährden. Der hier vorgeschlagene pragmatische Weg der Risikoquantifizierung lässt sich als Kompromiss zwischen Aufwand der Parametrisierung und praxistauglicher Umsetzbarkeit verstehen.

\subsection{Anwendung der risikogerechten Objektbewertung auf das Beispiel}

Für die Bestimmung des Risikopreises $\lambda_{\mathrm{T}}$ wurden ein nachhaltiger, risikoloser Zins von 3,0\% und eine (erwartete) Rendite der Alternativanlage eines empirischen Marktportfolios (Aktienindex) $E\left(\widetilde{r}_{m}\right)$ von 7,5\% $\%^{26}$ mit einer Standardabweichung $\sigma\left(\widetilde{r}_{m}\right)$ von $20 \%$, angesetzt. Wie in Abschn. 4.1 .4 erläutert wurde, ist für die hier gewählte Risikoprofilmethode der „Marktpreis des Risikos“ ( $\lambda$ ) über $T$ Perioden (Restnutzungsdauer) zu berechnen.

Wir nehmen also eine erwartete Marktrisikoprämie von 4,5\% (bei einem risikolosen Zinssatz von 3,0 \% sowie einer Marktrendite von 7,5\%) und eine Standardabweichung der Marktrendite von $20 \%$ an. Dies ergibt eine Sharpe-Ratio von $\lambda_{S \ddot{A}}=$ $22,5 \%$ bei einer einperiodigen Laufzeit und $\lambda_{S A ̈, T}=33,8 \%$ für den 60-periodigen Risikopreis. $^{27}$

Für die Bestimmung des Sicherheitsäquivalentes wird (wie bei Immobilienportfolios üblich) zunächst davon ausgegangen, dass aus Sicht des Bewertungssubjektes keine Diversifikation vorliegt $(d=1)$; Diversifikation auf Ebene des Portfolios wird indes explizit berücksichtigt. Tab. 4 fasst die Annahmen der Bewertung zusammen.

\subsection{Ermittlung des risikogerechten Wertes}

Abb. 5 fasst die Vorgehensweise ausgehend von den Annahmen und der darauf basierten Konstruktion der Szenarien bis hin zur Berechnung der Risikoabschläge gemäß der Gl. 5 zusammen. ${ }^{28}$

Aus der Simulation erhält man das Risikoprofil der freien Cashflows (nach Bodenwertverzinsung) (siehe Abb. 6).

\footnotetext{
${ }^{26}$ Die hier verwendeten Werte sind nominal und vor Steuern. Durch die Subtraktion des risikolosen Zinssatzes von der Marktrendite in Gl. 6 ist der Risikopreis unabhängig von der Inflation.

27 Berechnungsformel siehe Gl. 6 in Abschn. 4.1.4

28 Die unsichere Zahlungsreihe, kann eine beliebige bewertungsrelevante Zahlungsgröße sein, wie Free Cashflow, Cashflow, Ausschüttung, etc. Die Zahlung wird aus den (simulierten) unsicheren Jahresabschlüssen passend abgeleitet.
} 
Tab. 4 Annahmen für die risikogerechte Bewertung

\begin{tabular}{|c|c|}
\hline Bewertungsmethode & $\begin{array}{l}\text { Ertragswert mittels Sicherheitsäquivalenten (erweitert um Berücksich- } \\
\text { tigung der Zahlungsausfälle) mit Risikoprofilmethode }\end{array}$ \\
\hline Risikoinformationen & $\begin{array}{l}\text { Einschätzung zukünftiger Risiken der Zahlungen, die mittels Simulati- } \\
\text { on aggregiert werden }\end{array}$ \\
\hline Prognose der Zahlungsgröße & $\begin{array}{l}\text { 2-Phasen-Modell } \\
\text { 1. Phase: Detaillierte Planung (10 Jahre) } \\
\text { 2. Phase: Terminal Value (mit Wachstumsrate von 2,0\% für die freien } \\
\text { Cashflows, d. h. die erwartete Inflation wird ausgeglichen) }\end{array}$ \\
\hline Bewertungszeitpunkt & 31.12 .2014 \\
\hline Risikoloser Zinssatz & $3,0 \%$ (nachhaltig, bei $2 \%$ erwarteter Inflation) \\
\hline Marktrisikoprämie & $\begin{array}{l}\text { 4,5\% (nachhaltig, bei einem risikolosen Zinssatz von 3,0\% sowie } \\
\text { einer Marktrendite von 7,5\%) }\end{array}$ \\
\hline Risikomaß & Standardabweichung \\
\hline $\begin{array}{l}\text { Diversifikationseffekte des } \\
\text { Bewertungssubjekts (mit } \\
\text { portfolioexternen Objekten) }\end{array}$ & Zunächst keine portfolioexterne Diversifikation; $d=1$ \\
\hline $\begin{array}{l}\text { (Einjähriger/mehrjähriger) } \\
\text { Risikopreis } \lambda^{\mathrm{b}}\end{array}$ & $22,5 \% / 38,8 \%$ \\
\hline $\begin{array}{l}\text { Zinssatz für Bodenwertver- } \\
\text { zinsung }\end{array}$ & $\begin{array}{l}\text { Der Bodenwert wird gemäß Gutachten mit } 3,2 \% \text { verzinst und damit } \\
\text { als fast risikolos gesehen }\end{array}$ \\
\hline Bewertete Zahlung & Freie Cashflows abzgl. Bodenwertverzinsung \\
\hline Steuern & Nach Unternehmenssteuern \\
\hline Abschreibungsquote & Gemäß geplanter Abschreibung der einzelnen Gebäude \\
\hline Ausschüttung & $\begin{array}{l}\text { Keine Ausschüttung (ohne Berücksichtigung einer Ausschüttungspoli- } \\
\text { tik) }\end{array}$ \\
\hline Restnutzungsdauer & $\begin{array}{l}\text { Durchschnittliche (unsichere) Restnutzungsdauer mit Mittelwert } \\
60 \text { Jahre } \pm 6 \text { Jahre }\end{array}$ \\
\hline
\end{tabular}

${ }^{a}$ Typische langfristige mittlere Werte abgleitet aus dem deutschen Aktienmarktindex

${ }^{\mathrm{b}}$ Berechnungsformel siehe Gl. 6 in Abschn. 4.1.4

Durch die Nutzung der oben beschriebenen ergänzenden Methoden der Endwertverteilungs- und Risikoprofilmethode kann die Bewertung auf den freien Cashflow der Periode T abgestellt werden.

Der Mittelwert der Risikoprofilverteilung liegt bei 10,2 Mio. EUR, (das $95 \%$ Quantil liegt bei etwa 5,4 Mio. EUR). Die Standardabweichung beträgt 2,4 Mio. EUR. Der oben ermittelte Risikopreis (mehrjährig) beläuft sich auf $38,8 \%$. Somit folgt ein Wertbeitrag der Mieterträge in Höhe von 9,3 Mio. EUR.

$$
\begin{aligned}
W(\widetilde{Z})=E(\widetilde{Z})-\lambda_{T} \cdot d \cdot \sigma(\widetilde{Z}) & =10,2-0,388 \cdot 1 \cdot 2,4 \\
& =9,3 \text { Mio. EUR }
\end{aligned}
$$

Unter Berücksichtigung des als sicher angenommenen Bodenwertes erhält man den Wert der Immobilien in Höhe von 11,7 Mio. EUR. Sondervermögen wurden nicht unterstellt. 


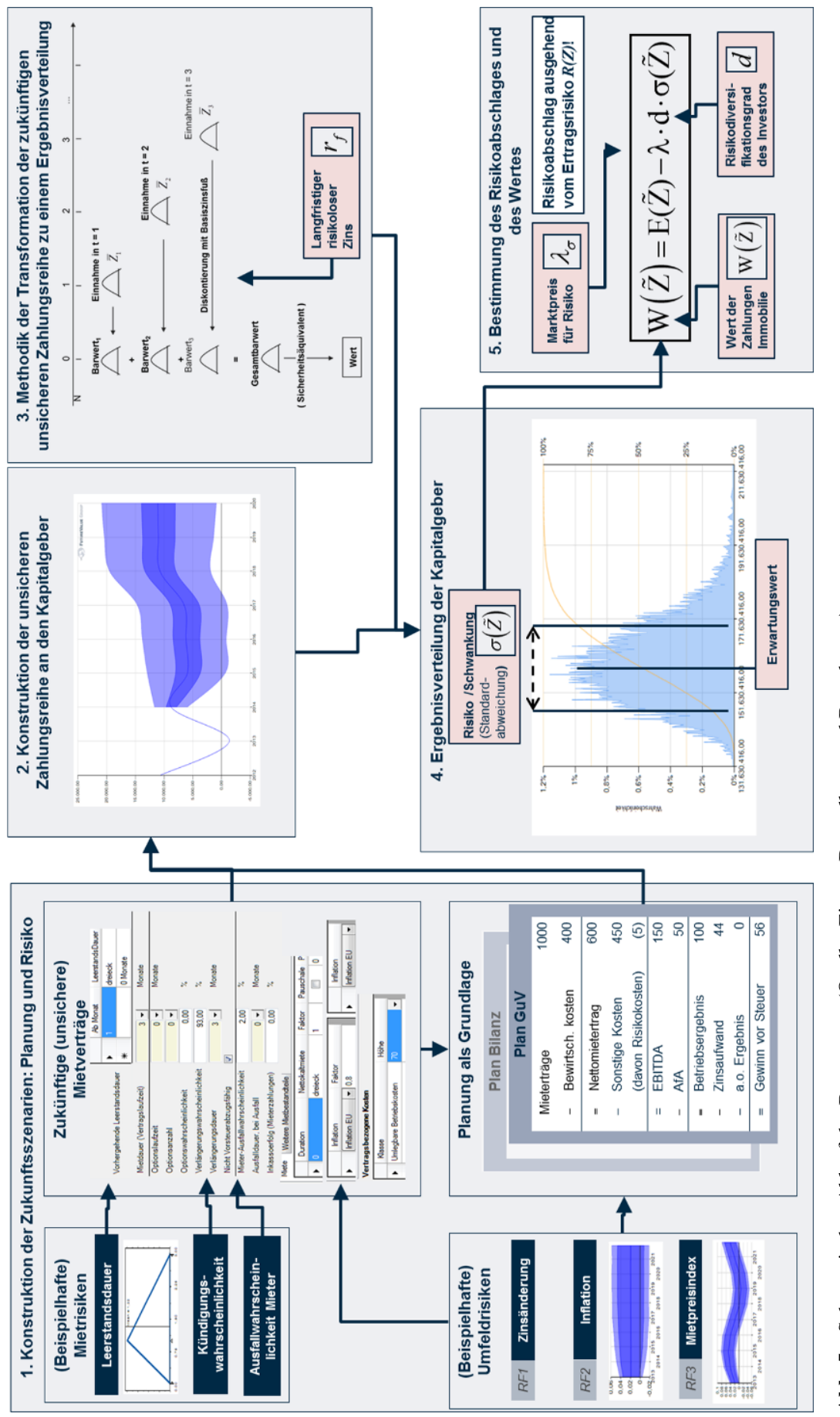

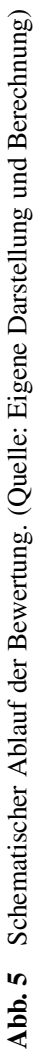




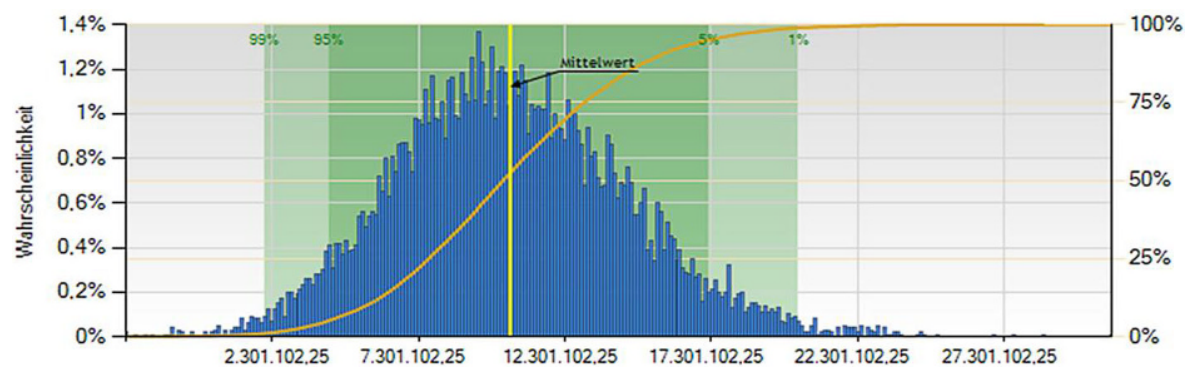

Abb. 6 Risikoprofilverteilung der freien Cashflows nach Bodenwertverzinsung (Quelle: Eigene Berechnung)

$$
\begin{aligned}
W(\operatorname{Immo})=W(\widetilde{Z})+W(\text { Boden }) & =9,3 \text { Mio. } €+2,4 \text { Mio. } € \\
& =11,7 \text { Mio. EUR }
\end{aligned}
$$

Dieser Wert entspricht einem Netto-Kaltmiete-Multiplikator von 19,3 bzw. einem Reinertragsmultiplikator von 28,8 (bezogen auf das Startjahr).

Der sehr hohe Wertunterschied zum Verkehrswert (gemäß Gutachten) in Höhe von 4,2 Mio. EUR kommt durch 4 Effekte zustande.

- Die langfristig prognostizierte Ertrags- und Kostenentwicklung (unter Berücksichtigung von Chancen und Gefahren) ist etwas günstiger als dies in den Verkehrswertgutachten angenommen wird („Chancen-Überhang“).

- Es gibt eine unterschiedliche Risikoeinschätzung des Bewertungssubjektes und des Marktes (abgebildet als Teil des Liegenschaftszinssatzes) bzgl. der einzelnen Objekte. Damit ist der risikogerechte Diskontierungszinssatz niedriger als der Liegenschaftszins.

- Es gibt Risikodiversifikationseffekte der Einzelobjekte innerhalb des Portfolios.

- Es gibt zeitliche Risikodiversifikationseffekte durch die Betrachtung der Risikoprofilverteilung als relevante Bewertungsgröße statt der einzelnen periodenbezogenen Risikobetrachtung wie bei der Berechnung des Liegenschaftszinssatzes (vgl. Abschn. 4.1.4).

Die einzelnen Effekte sind miteinander verbunden, wodurch die Summe der einzelnen Wirkungen nicht die Gesamtwirkung aller Effekte ergibt.

Das Beispiel basiert auf Marktdaten von 2014. In der Zwischenzeit sind die Immobilienpreise deutlich gestiegen. Durch diese Entwicklung läge auch in dem obigen Beispiel der Verkehrswert höher, wodurch ein Abbau der Fehlbewertung erfolgt.

\subsection{Zusammenfassende Antworten auf drei mögliche Fragen des Vorstands}

Mit diesem Ansatz lassen sich drei zentrale Fragen von Entscheidungsträgern im Immobilienunternehmen beantworten:

Erstens lässt sich zeigen, zu welchem Mindestpreis ein Objekt verkauft werden sollte (oder für welchen Maximalpreis gekauft werden sollte). Gerade in Phasen von 
Übertreibungen sollte man nicht nur den Marktpreis beachten, sondern auch jenen Wert, den dieses konkrete Objekt für den Akteur hat.

Zweitens ist es wichtig, die Portfolioeffekte von den Bewertungen der Einzelobjekte sauber zu trennen. Daraus lässt sich letztlich der Wert des Portfoliomanagements ermitteln.

Drittens, wie wertvoll sind diese Informationen zum Beispiel zur Verhandlung von Kreditkonditionen mit Finanzinstituten? Im Folgenden zeigen wir die Antworten auf diese drei Fragen anhand des gewählten Beispiels:

1. Preisuntergrenze: Welcher Preis muss auf dem Markt erzielt werden, damit ein Verkauf (ökonomisch) Sinn ergibt?

Im gewählten Beispiel bedeutet dies, dass unter Berücksichtigung des Ertrag-Risiko-Profils ein Verkauf des Immobilienportfolios erst oberhalb von 11,7 Mio. EUR, also deutlich oberhalb des Verkehrswerts von 7,5 Mio. EUR, ökonomisch sinnvoll ist, denn dies entspräche dem risikoadjustiertem Fundamentalwert.

2. Wertbeitrag einer sinnvollen Risikodiversifikation (auf Portfolioebene)

a. Direkte Wirkung: Wert des Portfolios (Eigenkapitalgebersicht)

Insgesamt lässt sich zeigen, dass der simulationsbasierte Ertragswert des Portfolios mit 11,7 Mio. EUR (inkl. Diversifikationseffekten zwischen den einzelnen Objekten des betrachteten Portfolios) deutlich über der Summe der Verkehrswerte der Einzelimmobilien gemäß dem Sachverständigengutachten von 7,5 Mio. EUR liegt (in dem definitionsgemäß diese Diversifikationseffekte nicht berücksichtigt werden). Diese Differenz hat mehrere Ursachen: Zum einen ist die langfristig prognostizierte Ertrags- und Kostenentwicklung (unter Berücksichtigung von Chancen und Gefahren) etwas günstiger als dies in den Verkehrswertgutachten angenommen wird („Chancen-Überhang“). Zum anderen ist der risikogerechte Diskontierungszinssatz niedriger als der Liegenschaftszins. Dies wiederum liegt daran, dass der Risikoumfang im Portfolio (in Relation zu den implizit erfassten Risiken des Liegenschaftszinses) relativ niedrig ist. Weiterhin betrachtet das Bewertungssubjekt die Risiken der Gesamterlöse (Risikoprofilmethode) und nicht jede Periodenschwankung als relevant. Schließlich wird in den Verkehrswertgutachten kein Risikodiversifikationseffekt auf Portfolioebene berücksichtigt.

b. Indirekte Wirkung: (z. B.) Vorteile bei der Fremdfinanzierung Zusätzlich zur Ermittlung des Wertes ließe sich z. B. eine Aussage zur möglichen Reduktion der Fremdkapitalkosten treffen: Es entsteht ein ökonomischer Mehrwert durch eine gezielte Portfoliostrukturierung innerhalb einer Immobiliengesellschaft. Die Möglichkeit der Darstellung der durch Risikodiversifikation entstandenen günstigen Risikosituation ist eine gute Grundlage, um mit den Kreditinstituten über eine Verbesserung von Fremdfinanzierungskonditionen zu sprechen. 


\section{Fazit}

Für die Praxis des Asset- und Portfoliomanagements bedeuten die Überlegungen dieses Beitrags, dass ergänzend zu dem existierenden gutachterlichen Verkehrswertverfahren - im Wesentlichen ein Preisschätzverfahren - risikobasierte fundamentale Bewertungsverfahren sinnvoll sind, um inhärente Risiken einer Immobilienanlage im heutigen Wert angemessen zu berücksichtigen.

Im Gegensatz zu den traditionellen Preisschätzverfahren wird der Diskontierungszinssatz bei den hier erläuterten Bewertungsverfahren explizit aus dem Risikoumfang der zukünftigen unsicheren Cashflows eines Assets abgeleitet. Dies ist charakteristisch für ein risikoorientiertes Bewertungsverfahren. Das Bewertungsergebnis ist damit vergleichsweise unabhängig von einer möglichen Fehleinschätzung durch Marktteilnehmer. Auf diesem Weg kann auch der Mehrwert eines Immobilienportfoliomanagements oder Assetmanagements berechnet werden. Dies ist keine Garantie, eine höhere Rendite zu erzielen. Aber die Wahrscheinlichkeit, Risiken systematisch ausgeblendet zu haben, ist deutlich reduziert worden.

Für die simulationsbasierte Bewertung sind im Vorfeld zahlreiche Annahmen zu treffen (z. B. über die Höchst- und Mindestmieten, mögliche Leerstandsrisiken etc.). Bei jeder Annahme können sich die Akteure irren, die Vorteile der simulationsbasierten Bewertung sind daher nicht, dass sie irrtumssicher ist, sondern dass sie erstens aufgrund der Berücksichtigung von Intervallen weniger irrtumsanfällig sind als Punktschätzungen und dass zweitens explizit Diversifikationseffekte in die Objektbewertung einfließen können. Daher entfaltet diese Bewertungsmethode ihre Stärken gerade im Nebeneinander mit der Ermittlung von Verkehrswerten. Für Marktakteure bleibt das Schätzen von aktuell realisierbaren Preisen weiterhin wichtig.

Mit den „Grundsätzen zur Bewertung von Immobilien“ (IDW S 10) hat das Institut der deutschen Wirtschaftsprüfer einen an den IDW S 1 angelehnten Immobilienbewertungsstandard veröffentlicht (s. hierzu u. a. Kleiber 2012). Dieser Standard basiert auf dem DCF-Ansatz, wobei gemäß IDW S 10 Tz. 63 bei der Bestimmung risikoadjustierter Diskontierungszinssätze ein Wahlrecht eingeräumt wird. Anstelle des im Allgemeinen vom IDW präferierten CAPM wird hier die auch in der Praxis präferierte Vorgehensweise einer ,komponentenweisen“ Ableitung mit einzelnen Risikozuschlägen erläutert. Die Komponenten sind dabei der risikolose Basiszinssatz $\left(r_{f}\right)$, ein allgemeiner immobilienmarktspezifischer Risikozuschlag sowie ein objektspezifischer Zuschlagssatz (vgl. Möller 2014). Das IDW unterstellt dabei implizit im Zeitverlauf konstante Risikozuschlagssätze (Möller 2014 sowie Creutzmann 2013). Möller (2014, S. 205) sieht den allgemeinen immobilienmarktspezifischen Risikozuschlag, den das IDW nicht spezifiziert, entsprechend des Standards „Discounted Cashflow-Bewertungsverfahren für Wohnimmobilien“ des Bundesverbandes deutscher Wohnungs- und Immobilienunternehmen in einer Bandbreite von 1,0-1,6\%. Im objektspezifischen Zuschlag sollen nun individuelle Objektfaktoren wie Lage, Eigenschaft und Zustand der Immobilie erfasst werden - wobei hier auf die an sich notwendigen Verfahren einer objektspezifischen Risikoanalyse zur Ableitung eines fundierten Risikozuschlagssatzes nicht eingegangen wird. 
Es ist erwähnenswert, dass im IDW S 10 - wie auch im IDW S 1 - eine ausdrückliche und konsequente Unterscheidung zwischen intrinsischem „Immobilienwert“ und einem geschätzten Verkehrswert nicht vorgenommen wird.

Investoren, die für Ihre Anlageentscheidungen auf stabile Cashflow-Renditen angewiesen sind, kommen nicht umhin, Bewertungsmodelle zu nutzen, die CashflowRisiken adäquat abbilden. In überhitzten Marktphasen werden ansonsten überbewertete Assets mit anderen überbewerteten Assets verglichen, was insbesondere für langfristig orientierte, risikoaverse Investoren zur Fehlallokation von Mitteln führt. Der risikogerechte Wert und der (geschätzte) aktuelle Preis (Verkehrswert) sind klar zu unterscheiden. Beides sind wesentliche Informationen. In diesem Beitrag wurde die Ermittlung des Wertes und damit die Eigenkapitalgebersicht ausführlich dargestellt. Hier soll nur als Ausblick erwähnt werden, dass mit leichter Erweiterung der skizzierten Vorgehensweise auch ein Rating, also die Beurteilung aus Fremdkapitalgebersicht, konsistent ableitbar ist.

Ziel dieser neuen Bewertung ist kein alternatives Preisschätzmodell in Konkurrenz zur Verkehrswertberechnung, sondern Ziel ist es, die Berechnung des Wertes eines Immobilien-Bewertungsobjektes aus Sicht eines Bewertungssubjektes basierend auf objekt- und subjektspezifischen Annahmen zu ermöglichen. Damit kann das Ziel auch nicht sein, Marktpreise besser zu treffen als dies von aktuellen Verkehrswertschätzungen gemacht wird, sondern den ,fundamentalen“ Wert einer Immobilie - auch innerhalb eines Portfolios - besser zu bestimmen und damit in Kombination von Marktpreisen bessere Entscheidungen bzgl. Kauf und Verkauf von Immobilien bzw. Portfoliostrukturierungen zu ermöglichen.

Interessenkonflikt W. Gleißner, T. Just und E. Kamarás geben an, dass kein Interessenkonflikt besteht.

Open Access Dieser Artikel wird unter der Creative Commons Namensnennung 4.0 International Lizenz (http://creativecommons.org/licenses/by/4.0/deed.de) veröffentlicht, welche die Nutzung, Vervielfältigung, Bearbeitung, Verbreitung und Wiedergabe in jeglichem Medium und Format erlaubt, sofern Sie den/die ursprünglichen Autor(en) und die Quelle ordnungsgemäß nennen, einen Link zur Creative Commons Lizenz beifügen und angeben, ob Änderungen vorgenommen wurden.

\section{Literatur}

Alkhatib H, Weitkamp A, Zaddach S, Neumann I (2015) Realistic uncertainty estimation of the market value based on a Fuzzy-Bayesian sales comparison approach. Proceedings of FIG working week, Sofia. (CD-Proceedings)

Amédée-Manesme C-O, Barthélémy F, Baroni M, Dupuy E (2013) Combining Monte Carlo simulations and options to manage the risk of real estate portfolios. J Prop Invest Finance 31(4):360-389

Artmann S, Finter P, Kempf A (2012) Determinants of expected stock returns: large sample evidence from the German market. J Bus Finance Account 39:758-784

Ballwieser W (1981) Die Wahl des Kalkulationszinsfußes bei der Unternehmensbewertung unter Berücksichtigung von Risiko und Geldentwertung. Betriebswirtsch Forsch Prax 33:97-114

Ballwieser W, Hachmeister D (2016) Unternehmensbewertung: Prozess, Methoden und Probleme, 5. Aufl. Schäffer-Poeschel, Stuttgart

Bamberg G, Dorfleitner G, Krapp M (2006) Unternehmensbewertung unter Unsicherheit: Zur entscheidungstheoretischen Fundierung der Risikoanalyse. Z Betriebswirtsch 76:287-307

Baroni M, Barthélémy F, Mokrane M (2007) Using rents and price dynamics in real estate portfolio valuation. Prop Manag 25(5):462-486 
Buch A, Dorfleitner G (2007) Ein Vergleich der Sicherheitsäquivalentmethode und der Risikoanalyse als Methoden zur Bewertung risikobehafteter Zahlungsströme. Z Betriebswirtsch 77:141-170

Chen L, Novy-Marx R, Zhang L (2011) An alternative Three-Factor model. Working paper. Washington University St. Louis. http://ssrn.com/abstract=1418117. Zugegriffen: 1. Juni 2017

Creutzmann A (2013) Net asset value in Theorie und Praxis. BewertungsPraktiker 8:64-69

French N, Gabrielli L (2005) Discounted cash flow: accounting for uncertainty. J Prop Invest Finance 22(6):101-119

Friedman M (1953) The Methodology of Positive Economics, in: Friedman M (Hrsg.) Essays in Positive Economics, Chicago/London, S. 3-43

Follain J, Giertz S (2011) Using Monte Carlo simulations to establish a new house price stress test. J Hous Econ 20(2):101-119

Gleißner W (2013) Unsicherheit, Risiko und Unternehmenswert. In: Petersen K, Zwirner C, Brösel G (Hrsg) Handbuch Unternehmensbewertung. Bundesanzeiger Verlag, Köln:691-721

Gleißner W (2017) Grundlagen des Risikomanagements im Unternehmen, 3. Aufl. Vahlen, München

Gleißner W, Wolfrum M (2008) Eigenkapitalkosten und die Bewertung nicht börsennotierter Unternehmen: Relevanz von Diversifikationsgrad und Risikomaß. Finanz Betr 9:602-614

Hagemeister M, Kempf A (2010) CAPM und erwartete Renditen. Betriebswirtschaft 70:145-164

Hanauer M, Kaserer C, Rapp MS (2013) Risikofaktoren und Multifaktormodelle für den Deutschen Aktienmarkt. Betriebswirtsch Forsch Prax 65:469-492

Hertz DB (1968) Investment policies that pay off. Harv Bus Rev 46:96-108

Hoesli M, Jani E, Bender A (2006) Monte Carlo simulations for real estate valuation. J Prop Invest Finance 24(2):102-122

Hughes WT (1995) Risk analysis and asset valuation: a Monte Carlo simulation using stochastic rents. J Real Estate Finance Econ 11:177-187

IDW (2012) Entwurf eines IDW Standards: Grundsätze zur Bewertung von Immobilien (IDW S 10). Wirtschaftsprüfung 5(Suppl):47

IDW (2013) IDW Standard: Grundsätze zur Bewertung von Immobilien (IDW S10). Wirtschaftsprüfung 6(Suppl):41

Jaffe AJ (1980) A note on the use of capitalization, discount, and forward rates. Appraisal J 48(1):24-30

Just T, Uttich S (2015) Es sind nicht nur Gebäude: Was Anleger über Immobilienmärkte wissen müssen. Frankfurter Societäts-Medien, Frankfurt

Kleiber W (2012) Marktwertermittlung nach ImmoWertV, 7. Aufl. Bundesanzeiger Verlag, Köln

Laux H, Schabel MM (2009) Subjektive Investitionsbewertung, Marktbewertung und Risikoteilung: Grenzpreise aus Sicht börsennotierter Unternehmen und individueller Investoren im Vergleich. Springer, Berlin

Meister D, Dressel K (2017) Valuation of real estate in Germany. In: Just T, Maennig W (Hrsg) Understanding German real estate markets, 2. Aufl. Springer, Berlin:133-145

Möller J (2014) Überlegungen zum Diskontierungszinssatz im IDW S10. Corp Finance 5:201-208

Pfnür A (2002) Performance-Controlling von Immobiliendirektinvestitionen. Z Immobilienökon 1(1):39-54

Pfnür A, Armonat S (2013) Modelling uncertain operational cash flows of real estate investments using simulations of stochastic processes. J Prop Invest Finance 31:481-501

Pohl P (2013) Value at Risk Analyse auf Basis einer stochastischen Unternehmensbewertung. Corp Finance 4:403-415

Pyhrr SA (1973) A Computer Simulation Model to Measure the Risk in Real Estate Investment. Real Estate Economics 1(1):48-78

Szumilo N, Gantenbein P, Gleißner W, Wiegelmann T (2016) Predicting uncertainty: the impact of risk measurement on value of real estate portfolios. J Prop Res 33(1):1-17

Walkshäusl C (2013) Fundamentalrisiken und Aktienrenditen - Auch hier gilt, mit weniger Risiko zu einer besseren Performance. Corp Finance 4:119-123

Wofford LE (1978) Simulation approach to the appraisal of income producing real estate. J Am Real Estate Urban Econ Assoc 6:370-394 